Universidad

BIBLIOTECA

Document downloaded from the institutional repository of the University of Alcala: http://ebuah.uah.es/dspace/

This is a postprint version of the following published document:

Lastra, A. \& Tahara, H., S. 2019, "Maillet type theorem for nonlinear totally characteristic partial differential equations", Mathematische Annalen, vol. 2019

Available at https://doi.org/10.1007/s00208-019-01864-x

C 2019 Springer

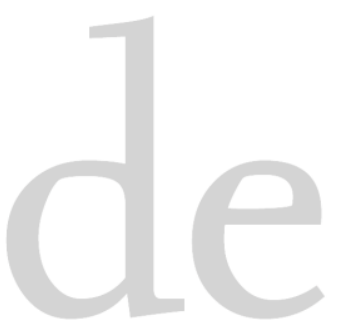

(Article begins on next page)

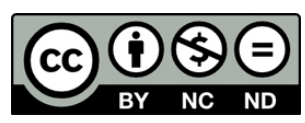

This work is licensed under a

Creative Commons Attribution-NonCommercial-NoDerivatives

4.0 International License. 


\title{
Maillet type theorem for nonlinear totally characteristic partial differential equations
}

\author{
Alberto Lastra ${ }^{1}$, Hidetoshi Tahara ${ }^{2}$ \\ ${ }^{1}$ University of Alcalá, Departamento de Física y Matemáticas, \\ Ap. de Correos 20, E-28871 Alcalá de Henares (Madrid), Spain, \\ alberto. lastra@uah.es \\ ${ }^{2}$ Sophia University, Department of Information and Communication Sciences, \\ Kioicho, Chiyoda-ku, Tokyo 102-8554, Japan. \\ h-tahara@sophia.ac.jp
}

\begin{abstract}
The paper discusses a holomorphic nonlinear singular partial differential equation $\left(t \partial_{t}\right)^{m} u=$ $F\left(t, x,\left\{\left(t \partial_{t}\right)^{j} \partial_{x}^{\alpha} u\right\}_{j+\alpha \leq m, j<m}\right)$ under the assumption that the equation is of nonlinear totally characteristic type. By using the Newton Polygon at $x=0$, the notion of the irregularity at $x=0$ of the equation is defined. In the case where the irregularity is greater than one, it is proved that every formal power series solution belongs to a suitable formal Gevrey class. The precise bound of the order of the formal Gevrey class is given, and the optimality of this bound is also proved in a generic case.
\end{abstract}

\section{Introduction}

In 1903, Maillet [16] showed that all formal power series solutions of nonlinear algebraic ordinary differential equations are in some formal Gevrey class (see Definition 1). This result was extended to general analytic ordinary differential equations by Malgrange [17]. In this paper, we achieve a Maillet type theorem for general nonlinear totally characteristic type partial differential equations.

We first fix some notations, used through the present work.

We write $\mathbb{N}=\{0,1,2, \ldots\}$ and $\mathbb{N}^{*}=\{1,2, \ldots\}$. For $m \in \mathbb{N}^{*}$, we consider the sets $I_{m}=\{(j, \alpha) \in$ $\mathbb{N} \times \mathbb{N} ; j+\alpha \leq m, j<m\}$, and $I_{m}(+)=\left\{(j, \alpha) \in I_{m} ; \alpha>0\right\}$. The pair $(t, x)$ stands for the variables in $\mathbb{C}_{t} \times \mathbb{C}_{x}$, and $\boldsymbol{z}=\left\{z_{j, \alpha}\right\}_{(j, \alpha) \in I_{m}}$ in $\mathbb{C}^{N}$ (with $N=\# I_{m}=m(m+3) / 2$ ).

$\mathbb{C}[[x]]$ denotes the ring of formal power series in $x$, and $\mathbb{C}[[t, x]]$ denotes the ring of formal power series in $(t, x)$. Similarly, $\mathbb{C}\{x\}$ denotes the ring of convergent power series in $x$, and $\mathbb{C}\{t, x\}$ denotes the ring of convergent power series in $(t, x)$.

Given $f(x)=\sum_{l>0} f_{l} x^{l} \in \mathbb{C}[[x]]$, we write $f(x) \gg 0$ if $f_{l} \geq 0$ for all $l \geq 0$ and $|f|(x)$ denotes the formal power series $\sum_{j \geq 0}\left|f_{j}\right| x^{j}$.

A. Lastra is partially supported by the project MTM2016-77642-C2-1-P of Ministerio de Economía y Competitividad, Spain. H. Tahara is partially supported by JSPS KAKENHI Grant. Number 15K04966. 
For $R>0$ we write $D_{R}=\{x \in \mathbb{C} ;|x|<R\}$, and $\bar{D}_{R}=\{x \in \mathbb{C} ;|x| \leq R\}$. We denote by $\mathcal{O}\left(D_{R}\right)$ the set of all holomorphic functions on $D_{R}$, and by $\mathcal{O}\left(\bar{D}_{R}\right)$ the set of all holomorphic functions in a neighborhood of $\bar{D}_{R}$.

Given $x \in \mathbb{R}$, we denote $[x]$ the integer part of $x$, and $[x]_{+}=\max \{x, 0\}$.

Let $F(t, x, \boldsymbol{z})$ be a function defined in a polydisk $\Delta$ centered at the origin of $\mathbb{C}_{t} \times \mathbb{C}_{x} \times \mathbb{C}_{\boldsymbol{z}}^{N}$. In this paper, we consider the following nonlinear partial differential equation

$$
\left(t \partial_{t}\right)^{m} u=F\left(t, x,\left\{\left(t \partial_{t}\right)^{j} \partial_{x}^{\alpha} u\right\}_{(j, \alpha) \in I_{m}}\right)
$$

under the assumptions

$\left.\mathrm{A}_{1}\right) F(t, x, \boldsymbol{z})$ is holomorphic in $\Delta$,

A $) F(0, x, \mathbf{0}) \equiv 0$ in $\Delta_{0}=\Delta \cap\{t=0, \boldsymbol{z}=\mathbf{0}\}$.

Under the previous assumptions, $F(t, x, \boldsymbol{z})$ can be expressed in the form

$$
F(t, x, \boldsymbol{z})=a(x) t+\sum_{(j, \alpha) \in I_{m}} b_{j, \alpha}(x) z_{j, \alpha}+R_{2}(t, x, \boldsymbol{z})
$$

where $R_{2}(t, x, \boldsymbol{z})$ is a holomorphic function on $\Delta$ whose Taylor expansion in $(t, \boldsymbol{z})$ has the form

$$
R_{2}(t, x, \boldsymbol{z})=\sum_{i+|\boldsymbol{\nu}| \geq 2} a_{i, \boldsymbol{\nu}}(x) t^{i} \boldsymbol{z}^{\boldsymbol{\nu}},
$$

where $\boldsymbol{\nu}=\left\{\nu_{j, \alpha}\right\}_{(j, \alpha) \in I_{m}} \in \mathbb{N}^{N},|\boldsymbol{\nu}|=\sum_{(j, \alpha) \in I_{m}} \nu_{j, \alpha}$ and $\boldsymbol{z}^{\boldsymbol{\nu}}=\prod_{(j, \alpha) \in I_{m}} z_{j, \alpha}{ }^{\nu_{j, \alpha}}$.

Different studies have been developed in the study of equation (1), which can be structured into three different blocks:

- Type 1: $b_{j, \alpha}(x) \equiv 0$ on $\Delta_{0}$ for any $(j, \alpha) \in I_{m}(+)$,

- Type $2: b_{j, \alpha}(0) \neq 0$ for some $(j, \alpha) \in I_{m}(+)$,

- Type 3: Cases not considered above.

Equation (1) under Type 1 condition deals with the so called nonlinear Fuchsian type partial differential equations. It has been studied by several authors such as Baouendi-Goulaouic [3], Gérard-Tahara [9, 10], Madi-Yoshino [15], Tahara-Yamazawa [23] and Tahara-Yamane [22]. A Gousart problem appears when considering equations within Type 2: Gérard-Tahara [11] discussed a particular class of equations in Type 2 and proved the existence of holomorphic solutions and also singular solutions of (1). An equation of the form (1) under the conditions in Type 3 is called a nonlinear totally characteristic type partial differential equation. The main thema of this paper is to discuss Type 3 under the following condition:

$\left.\mathrm{A}_{3}\right) b_{j, \alpha}(x)=O\left(x^{\alpha}\right)($ as $x \longrightarrow 0)$ for any $(j, \alpha) \in I_{m}(+)$.

Under this condition, we write $b_{j, \alpha}(x):=x^{\alpha} c_{j, \alpha}(x)$ for some holomorphic functions $c_{j, \alpha}(x)$ in a neighborhood of $x=0 \in \mathbb{C}$. We set

$$
\begin{aligned}
& C(x ; \lambda, \rho)=\lambda^{m}-\sum_{(j, \alpha) \in I_{m}} c_{j, \alpha}(x) \lambda^{j} \rho(\rho-1) \cdots(\rho-\alpha+1), \\
& L(\lambda, \rho)=C(0 ; \lambda, \rho) .
\end{aligned}
$$

Then, equation (1) is written in the form

$$
C\left(x ; t \partial_{t}, x \partial_{x}\right) u=a(x) t+R_{2}\left(t, x,\left\{\left(t \partial_{t}\right)^{j} \partial_{x}^{\alpha} u\right\}_{(j, \alpha) \in I_{m}}\right) .
$$


Proposition 1. Assume that the non-resonance condition

$$
L(k, l) \neq 0 \quad \text { for any }(k, l) \in \mathbb{N}^{*} \times \mathbb{N}
$$

is satisfied. Then, equation (1) admits a unique formal power series solution $u(t, x) \in \mathbb{C}[[t, x]]$, with $u(0, x) \equiv 0$.

About the convergence of this formal solution, nice results can be found in Chen-Tahara [8] and Tahara [21]. In the case where the formal solution is divergent, to measure the rate of divergence we use the following formal Gevrey classes:

Definition 1. (i) Let $s \geq 1, \sigma \geq 1$. We say that the formal series $f(t, x)=\sum_{k \geq 0, l \geq 0} a_{k, l} t^{k} x^{l} \in$ $\mathbb{C}[[t, x]]$ belongs to the formal Gevrey class $G\{t, x\}_{(s, \sigma)}$ of order $(s, \sigma)$ if the power series

$$
\sum_{k \geq 0, l \geq 0} \frac{a_{k, l}}{k !^{s-1} l !^{\sigma-1}} t^{k} x^{l}
$$

is convergent in a neighborhood of $(0,0) \in \mathbb{C}_{t} \times \mathbb{C}_{x}$.

(ii) Similarly, we say that the formal series $f(x)=\sum_{l \geq 0} a_{l} x^{l} \in \mathbb{C}[[x]]$ belongs to the formal Gevrey class $G\{x\}_{\sigma}$ of order $\sigma$ if the power series

$$
\sum_{l \geq 0} \frac{a_{l}}{l !^{\sigma-1}} x^{l}
$$

is convergent in a neighborhood of $0 \in \mathbb{C}_{x}$.

Let $u(t, x)$ be the formal solution of $(1)$, whose existence is guaranteed in Proposition 1, under condition $(\mathrm{N})$. The main aim in the present study is to answer the following natural questions:

a) Does $u(t, x)$ belong to $G\{t, x\}_{(s, \sigma)}$ for some $(s, \sigma)$ ?

b) If the answer is affirmative, determine the precise bound of the order $(s, \sigma)$.

In the case $m=1$, this problem was solved by Chen-Luo-Tahara [6]; in the case $m \geq 2$, Chen-Luo [5] has given a partial answer. The purpose of this paper is to give a final result in the general case.

The problem of finding exact Gevrey estimates attained to the formal solution of an equation is of great importance in the theory of summability of formal solutions to functional equations. In this concern, one can cite among others similar equations and problems which are studied recently: Chen-Luo [4], Shirai [19, 20] and Yamazawa [26]; Immink [13] in the study of difference equations; Di Vizio [24] on non linear q-difference equations; Zhang [27] on q-differencedifferential equations, Balser-Yoshino [2] when dealing with moment partial differential equations; Gontsov-Goryuchkina [12] in the framework of ODEs and in terms of generalized power series, Remy [18] in integro-differential equations. Optimality on the Gevrey bounds linked to formal solutions in the framework of dynamical systems, and its application to celestial mechanics can also be found in Baldomá-Fontich-Martín [1].

The paper is organized as follows. In Section 2, we describe the construction of the Newton polygon associated to the main equation, and related elements and properties. In Section 2.4, we state the two main results of the present work, namely Theorem 2, and Theorem 3. In Section 3, we present some preparatory discussions which are needed in the proof of (ii) of Theorem 2 . After that, in Section 4 we give a proof of (ii) of Theorem 2, and in Section 5 we give a proof of Theorem 3. In the last section, Section 6 , we give a slight generalization of the above results. 


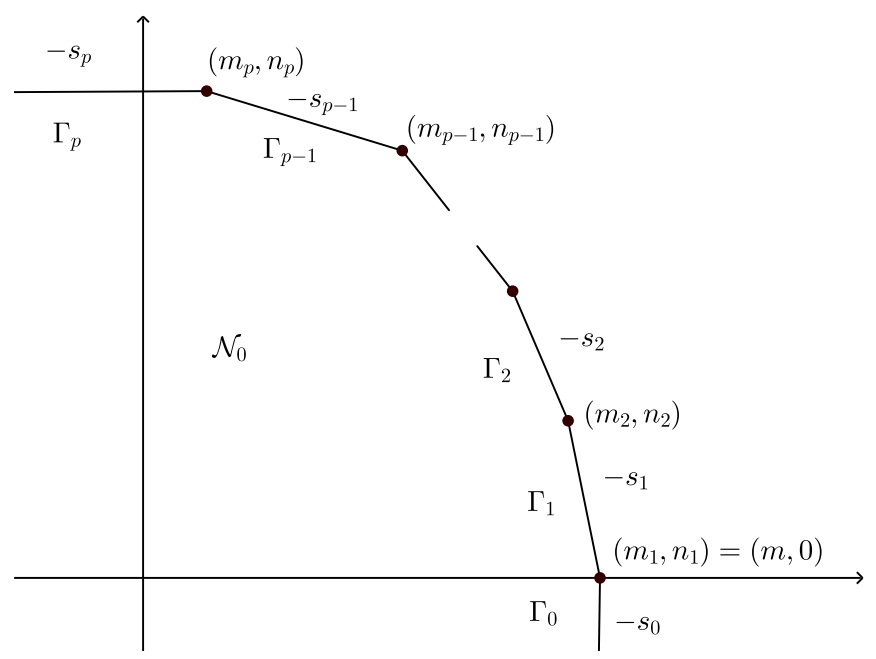

Figure 1: Newton polygon $\mathcal{N}_{0}$ at $x=0$

\section{Main result}

In this section, we first recall the definition of the Newton polygon $\mathcal{N}_{0}$ of equation (1) at $x=0$ and the generalized Poincaré condition (GP), in [21]. Then we define a notion of the irregularity $\sigma_{0}$ of (1) at $x=0$. After that, we give our main theorem, and the optimality of our condition.

\subsection{On the Newton polygon associated to the main equation}

Assume the conditions $\left.\left.\mathrm{A}_{1}\right), \mathrm{A}_{2}\right)$ and $\left.\mathrm{A}_{3}\right)$ hold, and define $c_{j, \alpha}(x)\left((j, \alpha) \in I_{m}\right)$ as in (3). Set $c_{m, 0}(x)=-1$, and

$$
\Lambda_{0}=\{(m, 0)\} \cup\left\{(j, \alpha) \in I_{m} ; c_{j, \alpha}(0) \neq 0\right\} .
$$

For $(a, b) \in \mathbb{R}^{2}$, we write $C(a, b)=\left\{(x, y) \in \mathbb{R}^{2} ; x \leq a, y \leq b\right\}$. Then, the Newton polygon $\mathcal{N}_{0}$ at $x=0$ of equation (1) is defined by the convex hull of the union of sets $C(j, \alpha)\left((j, \alpha) \in \Lambda_{0}\right)$ in $\mathbb{R}^{2}$; that is,

$$
\mathcal{N}_{0}=\text { the convex hull of } \bigcup_{(j, \alpha) \in \Lambda_{0}} C(j, \alpha)
$$

(see Section 2 in [21]). An example of Newton polygon is illustrated in Figure 1.

As is seen in Figure 1, the vertices of $\mathcal{N}_{0}$ consist of $p$ points

$$
\left(m_{1}, n_{1}\right)=(m, 0), \quad\left(m_{2}, n_{2}\right), \cdots, \quad\left(m_{p-1}, n_{p-1}\right), \quad\left(m_{p}, n_{p}\right),
$$

and the boundary of $\mathcal{N}_{0}$ consists of a vertical half line $\Gamma_{0},(p-1)$-segments $\Gamma_{1}, \Gamma_{2}, \ldots, \Gamma_{p-1}$, and a horizontal half line $\Gamma_{p}$. We denote the slope of $\Gamma_{i}$ by $-s_{i}(i=0,1,2, \ldots, p)$, and have

$$
s_{0}=\infty>s_{1}>s_{2}>\cdots>s_{p-1}>s_{p}=0 .
$$

Let us recall the following definition (see Definition 1 in [21]). 
Definition 2. We say that equation (1) has a regular singularity at $x=0$ if the following condition is satisfied:

$$
\text { if } c_{j, \alpha}(0)=0 \text { and } c_{j, \alpha}(x) \not \equiv 0 \text {, then }(j, \alpha) \in \mathcal{N}_{0} \text {. }
$$

Otherwise, that is, if $(R)$ is not satisfied then we say that equation (1) has an irregular singularity at $x=0$.

\subsection{Generalized Poincaré condition}

For $1 \leq i \leq p-1$ we define the characteristic polynomial on $\Gamma_{i}$ by

$$
P_{i}(X)=\sum_{(j, \alpha) \in \Lambda_{0} \cap \Gamma_{i}} c_{j, \alpha}(0) X^{j-m_{i+1}}=c_{m_{i}, n_{i}}(0) X^{m_{i}-m_{i+1}}+\cdots+c_{m_{i+1}, n_{i+1}}(0) .
$$

We denote $\lambda_{i, h}\left(1 \leq h \leq m_{i}-m_{i+1}\right)$ the roots of $P_{i}(X)=0$ which are called the characteristic roots on $\Gamma_{i}$. In the case $i=p$, the characteristic polynomial on $\Gamma_{p}$ is defined by $P_{p}(X)=1$ if $m_{p}=0$, and by

$$
P_{p}(X)=\sum_{(j, \alpha) \in \Lambda_{0} \cap \Gamma_{p}} c_{j, \alpha}(0) X^{j}=c_{m_{p}, n_{p}}(0) X^{m_{p}}+\cdots, \quad \text { if } m_{p} \geq 1 .
$$

In the case $m_{p} \geq 1$, the roots $\lambda_{p, h}\left(1 \leq h \leq m_{p}\right)$ of $P_{p}(X)=0$ are called the characteristic roots on $\Gamma_{p}$. We define the generalized Poincaré condition in the following way:

(GP)(Generalized Poincaré condition)

(i) $\lambda_{i, h} \in \mathbb{C} \backslash[0, \infty)$ for all $1 \leq i \leq p-1$ and $1 \leq h \leq m_{i}-m_{i+1}$,

(ii) $\lambda_{p, h} \in \mathbb{C} \backslash \mathbb{N}^{*}$ for $1 \leq h \leq m_{p}$.

Remark. For $p=1$, we have $\mathcal{N}_{0}=\left\{(x, y) \in \mathbb{R}^{2} ; x \leq m, y \leq 0\right\}$. Therefore, (GP) is reduced to its second statement, and (GP) is equivalent to $(\mathrm{N})$.

We set

$$
\phi(\lambda, \rho)=\sum_{i=1}^{p} \lambda^{m_{i}} \rho^{n_{i}}=\lambda^{m}+\lambda^{m_{2}} \rho^{n_{2}}+\cdots+\lambda^{m_{p}} \rho^{n_{p}} .
$$

The following results can be found in Proposition 1 and Theorem 2, [21], respectively.

Lemma 1. The following two conditions are equivalent:

- (N) and (GP) hold.

- There exists $c_{0}>0$ such that

$$
|L(k, l)| \geq c_{0} \phi(k, l)
$$

for every $(k, l) \in \mathbb{N}^{*} \times \mathbb{N}$.

Theorem 1 (Theorem 2 in [21]). If (N), (R) and (GP) hold, the unique formal power series solution in Proposition 1 is convergent in a neighborhood of $(0,0) \in \mathbb{C}_{t} \times \mathbb{C}_{x}$. 


\subsection{On the irregularity at $x=0$}

We set $\Lambda=\left\{(j, \alpha) \in I_{m} ; c_{j, \alpha}(x) \not \equiv 0\right\}$ and $\Lambda_{1}=\left\{(j, \alpha) \in I_{m} ; c_{j, \alpha}(0)=0, c_{j, \alpha}(x) \not \equiv 0\right\}$. It holds that $\Lambda \cup\{(m, 0)\}=\Lambda_{0} \cup \Lambda_{1}$. For $(j, \alpha) \in \Lambda_{1}$ we define

$$
\begin{aligned}
p_{j, \alpha} & =\text { the order of the zeros of } c_{j, \alpha}(x) \text { at } x=0 \quad(\geq 1), \\
d_{j, \alpha} & =\min \left\{y \in \mathbb{R} ;(j, \alpha-y) \in \mathcal{N}_{0}\right\} .
\end{aligned}
$$

Observe that $p_{j, \alpha} \geq 1$ and $d_{j, \alpha}$ is well-defined, for every $(j, \alpha) \in \Lambda_{1}$. Moreover, one has $(j, \alpha) \in$ $\mathcal{N}_{0}$ if and only if $d_{j, \alpha} \leq 0$. We define the irregularity $\sigma_{0}$ at $x=0$ of (1) by

$$
\sigma_{0}=\max \left[1, \max _{(j, \alpha) \in \Lambda_{1}} \frac{p_{j, \alpha}+d_{j, \alpha}}{p_{j, \alpha}}\right] .
$$

The reason why we call this "the irregularity at $x=0$ " is explained by the following lemma:

Lemma 2. The regular singularity condition ( $R$ ) (see Definition 2) is satisfied if and only if $\sigma_{0}=1$.

\subsection{Main results}

Given a formal power series $f(t, \boldsymbol{z})=\sum_{i+|\boldsymbol{\nu}| \geq 0} f_{i, \boldsymbol{\nu}} t^{i} \boldsymbol{z}^{\boldsymbol{\nu}} \in \mathbb{C}[[t, \boldsymbol{z}]]$, we define the valuation $\operatorname{val}(f)$ of $f(t, \boldsymbol{z})$ by

$$
\operatorname{val}(f)=\min \left\{i+|\boldsymbol{\nu}| ; f_{i, \boldsymbol{\nu}} \neq 0\right\} .
$$

If $f(t, \boldsymbol{z}) \equiv 0$ we set $\operatorname{val}(f)=\infty$. The previous definition is naturally extended to a holomorphic function defined in a neighborhood of $(0,0) \in \mathbb{C}_{t} \times \mathbb{C}_{z}^{N}$ by means of its Taylor expansion at the origin.

Let $\sigma_{0}$ be the irregularity at $x=0$ of $(1)$, and let $R_{2}(t, x, \boldsymbol{z})$ be as in (2). We put

$$
L_{\mu, j, \alpha}=\operatorname{val}\left(\left(\partial_{z_{j, \alpha}} \partial_{x}^{\mu} R_{2}\right)(t, 0, \boldsymbol{z})\right), \quad \mu \in \mathbb{N}, \quad(j, \alpha) \in I_{m}
$$

and set

$$
s_{0}=1+\max \left[0, \max _{0 \leq \mu<m}\left(\max _{(j, \alpha) \in I_{m}} \frac{j+\mu+\sigma_{0}(\alpha-\mu)-m}{L_{\mu, j, \alpha}}\right)\right] .
$$

Theorem 2 (Main Theorem). Assume the conditions $\left.\left.\left.A_{1}\right), A_{2}\right), A_{3}\right),(N)$ and $(G P)$ hold. Let $u(t, x) \in \mathbb{C}[[t, x]]$ be the unique formal solution of (1) satisfying $u(0, x) \equiv 0$. Then, the following results hold:

(i) If $\sigma_{0}=1$, then $u(t, x)$ is convergent in a neighborhood of $(0,0) \in \mathbb{C}_{t} \times \mathbb{C}_{x}$.

(ii) If $\sigma_{0}>1$, then $u(t, x) \in G\{t, x\}_{(s, \sigma)}$ for any $s \geq s_{0}$ and $\sigma \geq \sigma_{0}$.

Since $\sigma_{0}=1$ is equivalent to condition (R) (see Lemma 2), the first part in the previous result is a known fact, which can be found in Theorem 1. In the case $m=1$, the second statement of the previous result was proved by H. Chen, Z. Luo and the second author [6]. The proof of such statement under general settings is put forward in Section 4. It is worth mentioning that indices close to $\sigma_{0}$ and $s_{0}$ are defined in the work by H. Chen and Z. Luo [5].

For $0 \leq \mu<m$ we set

$$
I_{m, \mu}=\left\{(j, \alpha) \in I_{m} ; \alpha>\mu,\left(\partial_{z_{j, \alpha}} \partial_{x}^{\mu} R_{2}\right)(t, 0, \boldsymbol{z}) \not \equiv 0\right\} .
$$

The next result is a direct consequence of Theorem 2 . 
Corollary 1. Under the assumption

$$
\sigma_{0} \leq \frac{m-j-\mu}{\alpha-\mu} \quad \text { for } 0 \leq \mu<m \text { and }(j, \alpha) \in I_{m, \mu}
$$

one has $u(t, x) \in G\{t, x\}_{\left(1, \sigma_{0}\right)}$.

Corollary 1 implies that the unique formal power series solution $u(t, x)$ of $(1)$ is holomorphic in the variable $t$.

Let $a_{i, \boldsymbol{\nu}}(x)$, with $i+|\boldsymbol{\nu}| \geq 2$, be as in (2), and set

$$
\mathcal{L}=\left\{(i, \boldsymbol{\nu}): i+|\boldsymbol{\nu}| \geq 2,|\boldsymbol{\nu}| \geq 1, a_{i, \boldsymbol{\nu}}(x) \not \equiv 0\right\} .
$$

The following theorem asserts that our condition in Theorem 2 is optimal in a generic case.

Theorem 3 (Optimality). Assume the conditions $\left.\left.A_{1}\right), A_{2}\right)$ and $\left.A_{3}\right)$ hold. In addition to that, we adopt $\mathcal{L} \neq \emptyset$, and also the following conditions:

$\left.c_{1}\right)\left(\partial_{x}^{\mu} a\right)(0)>0$ for $0 \leq \mu \leq m$ and $a(x) \gg 0$,

$\left.c_{2}\right) c_{j, \alpha}(0) \leq 0$ for every $(j, \alpha) \in I_{m}$,

$\left.c_{3}\right) c_{j, \alpha}(x)-c_{j, \alpha}(0) \gg 0$ for every $(j, \alpha) \in I_{m}$,

$\left.c_{4}\right) a_{i, \boldsymbol{\nu}}(x) \gg 0$ for all $(i, \boldsymbol{\nu}) \in \mathbb{N} \times \mathbb{N}^{N}$ with $i+|\boldsymbol{\nu}| \geq 2$.

Then, equation (1) has a unique formal solution $u(t, x) \in \mathbb{C}[[t, x]]$ satisfying $u(0, x) \equiv 0$. Moreover, $u(t, x) \in G\{t, x\}_{(s, \sigma)}$ if and only if $(s, \sigma)$ is such that $s \geq s_{0}$ and $\sigma \geq \sigma_{0}$.

In view of the previous result, we may say that the index $\left(s_{0}, \sigma_{0}\right)$ defined in $(8)$ and $(9)$ is the formal Gevrey index of the equation (1). The proof of Theorem 3 is given in detail in Section 5.

Remark. The condition $\mathcal{L} \neq \emptyset$ is essential to the optimality on Theorem 3. In the case $\mathcal{L}=\emptyset$, the optimality of the index $\sigma_{0}$ is a very delicate problem, as is seen in the following example.

(1) In the case

$$
\left(\left(t \partial_{t}\right)^{4}+\left(x \partial_{x}\right)^{2}\right) u=\frac{t}{1-x}+x\left(t \partial_{t}\right)^{2}\left(x \partial_{x}\right)^{2} u,
$$

we have $\mathcal{L}=\emptyset$ and $\sigma_{0}=2$. However, it is straight to check that the unique formal solution of such equation has the form $u(t, x)=t u_{1}(x)$, and it is convergent in a neighborhood of $x=0$. This shows that $\sigma_{0}=2$ is not optimal.

(2) On the other hand, if we consider the equation

$$
\left(\left(t \partial_{t}\right)^{4}+\left(x \partial_{x}\right)^{2}\right) u=\frac{x t}{1-t}+x\left(t \partial_{t}\right)^{2}\left(x \partial_{x}\right)^{2} u,
$$

the unique formal solution is given by

$$
u(t, x)=\sum_{k \geq 1} \sum_{l \geq 1} \frac{k^{2(l-1)}(l-1) !^{2}}{\left(k^{4}+l^{2}\right) \cdots\left(k^{4}+2^{2}\right)\left(k^{4}+1^{2}\right)} t^{k} x^{l} .
$$

Here, $u(t, x) \in G\{t, x\}_{(s, \sigma)}$ if and only if $s \geq 1$ and $\sigma \geq 2$ (the necessity is verified by looking at the summation over $\left.\left\{(k, l): k=\left[l^{1 / 2}\right], l \in \mathbb{N}^{\star}\right\}\right)$. In this case, $\sigma_{0}=2$ is optimal. 
Example: We consider the equation

$$
\left(\left(t \partial_{t}\right)^{4}+\left(x \partial_{x}\right)^{2}\right) u=a(x) t+x\left(t \partial_{t}\right)^{2}\left(x \partial_{x}\right)^{2} u+x^{\mu} t^{i}\left(\left(t \partial_{t}\right)^{j} \partial_{x}^{\alpha} u\right)^{n},
$$

where $a(x) \in \mathbb{C}\{x\},(j, \alpha) \in I_{4}$, and $\mu, i, n \in \mathbb{N}$ with $i+n \geq 2$ and $n \geq 1$. Suppose the conditions $\left(\partial_{x} a\right)(0)>0,\left(\partial_{x}^{\alpha} a\right)(0)>0$ (only in the case $n \geq 2$ ), and $a(x) \gg 0$ hold. Then we have:

- $\sigma_{0}=2$ and $s_{0}=1+\max \left[0, \frac{j+2 \alpha-\mu-4}{i+n-1}\right]$.

- The equation (11) has a unique formal solution $u(t, x) \in \mathbb{C}[[t, x]]$ satisfying $u(0, x) \equiv 0$, and it belongs to the clsass $G\{t, x\}_{(s, \sigma)}$ if and only if $s \geq s_{0}$ and $\sigma \geq 2$.

- The formal solution $u(t, x)$ belongs to $G\{t, x\}_{(1,2)}$, if and only if one of the following conditions 1) 5) are satisfied:

$$
\begin{aligned}
& \text { 1) } \mu \geq 4 \text {, } \\
& \text { 2) } \mu=3 \text { and } \alpha \leq 3 \text {, } \\
& \text { 3) } \mu=2 \text { and }(j, \alpha) \in\left\{(k, \beta) \in I_{4} ; \beta \leq 2\right\} \cup\{(0,3)\} \text {, } \\
& \text { 4) } \mu=1 \text { and }(j, \alpha) \in\left\{(k, \beta) \in I_{4} ; \beta \leq 1\right\} \cup\{(0,2),(1,2)\} \text {, } \\
& \text { 5) } \mu=0 \text { and }(j, \alpha) \in\left\{(k, \beta) \in I_{4} ; k+\beta \leq 2\right\} \cup\{(2,1),(3,0)\} \text {. }
\end{aligned}
$$

Example: Let us consider

$$
t \partial_{t} u=(1+x) t+x^{p}\left(x \partial_{x}\right) u+x^{\mu} t^{i} u^{m}\left(\partial_{x} u\right)^{n}
$$

where $p, \mu, i, m, n \in \mathbb{N}$, with $p, \mu, n \geq 1$, and $i+m+n \geq 2$. It is straight to check that (12) has a unique formal solution $u(t, x) \in \mathbb{C}[[t, x]]$ which satisfies $u(0, x) \equiv 0$.

(1) By Theorem 3 (or Example 2.4 in [6]) we see that $u(t, x) \in G\{t, x\}_{\left(1, \sigma_{0}\right)}$, for $\sigma_{0}=1+1 / p$.

(2) In addition to this, by the results in Chen-Luo-Zhang [7] we derive the following result: if $\mu \geq p+1$, then the formal solution $u(t, x)$ is $p$-summable in any direction $d \in[0,2 \pi) \backslash S$ with $S=\{2 \pi k / p: k=0,1, \ldots, p-1\}$ in the $x$-variable.

In the case that $m=1$, the summability of formal solutions of nonlinear totally characteristic equations is studied by [7] and Luo-Chen-Zhang [14]. In the general case, this question is still open. Thus, the next target of our research should be to solve the following problem: in the general case, find appropriate conditions under which the summability of the formal solution can be attained.

\section{Some preparatory discussions}

In this section, we present some preparatory discussions which are needed in the proof of (ii) of Theorem 2.

Let $m \in \mathbb{N}, \sigma \geq 1$ and $f(x)=\sum_{j \geq 0} f_{j} x^{j} \in \mathbb{C}[[x]]$. In [6], the authors make use of the formal Borel operator $\mathcal{B}_{\sigma}$ defined by

$$
\mathcal{B}_{\sigma}[f](x)=\sum_{j \geq 0} \frac{f_{j}}{j^{\sigma-1}} x^{j}
$$


in order to achieve a Maillet-type result. In this paper, we need a refinement. For this purpose, we define the operator $\mathcal{B}_{\sigma}^{(m)}$ by

$$
\mathcal{B}_{\sigma}^{(m)}[f](x)=f_{0}+f_{1} x+\cdots+f_{m-1} x^{m-1}+\sum_{j \geq m} \frac{f_{j}}{(j-m) !^{\sigma-1}} x^{j}=\sum_{j \geq 0} \frac{f_{j}}{[j-m]_{+} !^{\sigma-1}} x^{j} .
$$

Lemma 3. Let $f(x), g(x) \in \mathbb{C}[[x]]$. We also take $\sigma \geq 1$ and $m \in \mathbb{N}$. The following statements hold:

$$
\begin{aligned}
& \mathcal{B}_{\sigma}[|f|](x)=\mathcal{B}_{\sigma}^{(0)}[|f|](x) \ll \mathcal{B}_{\sigma}^{(1)}[|f|](x) \ll \mathcal{B}_{\sigma}^{(2)}[|f|](x) \ll \ldots ; \\
& \mathcal{B}_{\sigma}^{(m)}[f g](x) \ll \mathcal{B}_{\sigma}^{(m)}[|f|](x) \times \mathcal{B}_{\sigma}^{(m)}[|g|](x) ; \\
& \mathcal{B}_{\sigma}^{(m)}\left[x^{k} f\right](x)=x^{k} \mathcal{B}_{\sigma}^{(m-k)}[f](x) \quad \text { for } 1 \leq k \leq m .
\end{aligned}
$$

The proof of Lemma 3 is straightforward.

A Nagumo-like result is also derived, which will be useful in the sequel.

Lemma 4. Let $m \in \mathbb{N}^{*}$ and $0<R \leq 1$. Suppose that $f(x) \in \mathbb{C}[[x]]$ satisfies

$$
\mathcal{B}_{\sigma}^{(m)}[|f|](x) \ll \frac{C}{(R-x)^{a}}
$$

for some $C>0$ and $a \geq 1$. Then, it holds that

$$
\mathcal{B}_{\sigma}^{(m-1)}\left[\partial_{x}|f|\right](x) \ll \frac{a C}{(R-x)^{a+1}}, \quad \mathcal{B}_{\sigma}^{(m)}\left[\partial_{x}|f|\right](x) \ll \frac{(a+\sigma)^{\sigma} e^{\sigma} C}{(R-x)^{a+\sigma}} .
$$

Proof. We write $f(x)=\sum_{j \geq 0} f_{j} x^{j}$. By the assumption (13) we have

$$
\begin{cases}\left|f_{j}\right| \leq \frac{C}{R^{a+j}} \frac{a(a+1) \cdots(a+j-1)}{j !}, & \text { if } 0 \leq j \leq m-1, \\ \frac{\left|f_{j}\right|}{(j-m) !^{\sigma-1}} \leq \frac{C}{R^{a+j}} \frac{a(a+1) \cdots(a+j-1)}{j !}, & \text { if } j \geq m .\end{cases}
$$

These estimates yield

$$
\mathcal{B}_{\sigma}^{(m-1)}\left[\partial_{x}|f|\right](x) \ll \sum_{j \geq 0} \frac{a C}{R^{a+1+j}} \frac{(a+1) \cdots(a+j)}{j !} x^{j}=\frac{a C}{(R-x)^{a+1}},
$$

which proves the first statement of Lemma 4 . The second follows from the next estimates:

$$
\begin{aligned}
\mathcal{B}_{\sigma}^{(m)}\left[\partial_{x}|f|\right](x) \ll & \sum_{0 \leq j \leq m-1}(j+1) \frac{C}{R^{a+j+1}} \frac{a(a+1) \cdots(a+j)}{(j+1) !} x^{j} \\
& +\sum_{j \geq m} \frac{(j+1)(j+1-m) !^{\sigma-1}}{(j-m) !^{\sigma-1}} \frac{C}{R^{a+j+1}} \frac{a(a+1) \cdots(a+j)}{(j+1) !} x^{j} \\
& \ll \sum_{j \geq 0} \frac{(j+1)^{\sigma-1} C}{R^{a+j+1}} \frac{a(a+1) \cdots(a+j)}{j !} x^{j} .
\end{aligned}
$$

Here, we have used that $1 \leq(j+1)($ for $0 \leq j \leq m-1)$ and $(j+1-m) \leq(j+1)$ (for $j \geq m)$. 
Let $A=(a+\sigma)^{\sigma} e^{\sigma}$. Since

$$
\frac{(a+\sigma)^{\sigma} e^{\sigma} C}{(R-x)^{a+\sigma}}=\sum_{j \geq 0} \frac{A C}{R^{a+\sigma+j}} \frac{(a+\sigma)(a+\sigma+1) \cdots(a+\sigma+j-1)}{j !} x^{j},
$$

and $R^{a+\sigma+j} \leq R^{a+j+1}$, the proof is concluded after checking that

$$
\frac{(j+1)^{\sigma-1} \Gamma(a+j+1) \Gamma(a+\sigma)}{\Gamma(a) \Gamma(a+\sigma+j)} \leq A .
$$

We refer to the proof of Lemma 5 in [6] for a detailed demonstration of such estimate.

Corollary 2. Let $m \in \mathbb{N}^{*}$ and $0<R \leq 1$. Suppose that $f(x) \in \mathbb{C}[[x]]$ satisfies (13). Then, for all $1 \leq \mu \leq m$ and $k \geq 1$ we have

$$
\begin{aligned}
& \mathcal{B}_{\sigma}^{(m-\mu)}\left[\partial_{x}^{\mu}|f|\right](x) \ll \frac{a(a+1) \cdots(a+\mu-1) C}{(R-x)^{a+\mu}}, \\
& \mathcal{B}_{\sigma}^{(m-\mu)}\left[\partial_{x}^{k+\mu}|f|\right](x) \ll \frac{a(a+1) \cdots(a+\mu-1) A_{\mu, k} C}{(R-x)^{a+\mu+k \sigma}},
\end{aligned}
$$

where $A_{\mu, k}=e^{k \sigma} \prod_{h=1}^{k}(a+\mu+h \sigma)^{\sigma}$.

\subsection{On the Newton polygon}

Let $\mathcal{N}_{0}$ be the Newton polygon associated to equation (1), and let $\phi(\lambda, \rho)$ be as in (6). For $(j, \alpha) \in I_{m}$ with $(j, \alpha) \notin \mathcal{N}_{0}$ we recall that

$$
d_{j, \alpha}=\min _{(j, x) \in \mathcal{N}_{0}}|\alpha-x|=\min \left\{y \in \mathbb{R} ;(j, \alpha-y) \in \mathcal{N}_{0}\right\} .
$$

Proposition 2. Let $(j, \alpha) \in I_{m}$. The following results hold.

(i) If $(j, \alpha) \in \mathcal{N}_{0}$, we have

$$
k^{j} l^{\alpha} \leq \phi(k, l), \quad(k, l) \in \mathbb{N}^{*} \times \mathbb{N} .
$$

(ii) If $(j, \alpha) \notin \mathcal{N}_{0}$, for $p \geq 1$ and $\sigma \geq 1+d_{j, \alpha} / p$ we have

$$
\frac{k^{j}(l-p)^{\alpha}}{\phi(k, l)} \leq\left(\frac{l !}{(l-p) !}\right)^{\sigma-1}, \quad \text { for }(k, l) \in \mathbb{N}^{*} \times \mathbb{N} \text { with } l \geq p .
$$

Proof. Part (i) is proved in Lemma 7 [21]. Part (ii) follows from Lemma 5 and Lemma 6 below.

Lemma 5. Let $m, n, p \in \mathbb{N}$ with $m \geq 1, n \geq 0$ and $p \geq 1$. Suppose that $0 \leq j<m$ and $\alpha>n(m-j) / m$. Set $d=\alpha-n(m-j) / m$. Then, if $\sigma \geq(p+d) / p$ holds, we have

$$
\frac{k^{j}(l-p)^{\alpha}}{k^{m}+l^{n}} \leq\left(\frac{l !}{(l-p) !}\right)^{\sigma-1}, \quad(k, l) \in \mathbb{N}^{*} \times \mathbb{N} \text { with } l \geq p .
$$

The geometry described in Lemma 5 is illustrated in Figure 2. 


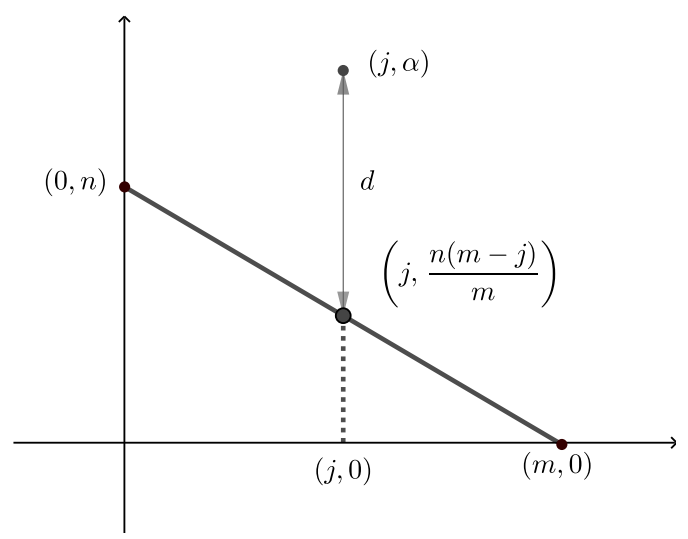

Figure 2: Geometry of the result described in Lemma 5

Proof. If $j=0$ we have $d=\alpha-n>0$ and since $\sigma \geq(p+d) / p$ we have

$$
\frac{k^{j}(l-p)^{\alpha}}{k^{m}+l^{n}}=\frac{(l-p)^{\alpha}}{k^{m}+l^{n}} \leq(l-p)^{\alpha-n}=(l-p)^{d} \leq\left(\frac{l !}{(l-p) !}\right)^{\sigma-1},
$$

which yields (14).

If $n=0$, we have $d=\alpha$ and (14) follows from

$$
\frac{k^{j}(l-p)^{\alpha}}{k^{m}+l^{n}}=\frac{k^{j}(l-p)^{\alpha}}{k^{m}} \leq(l-p)^{\alpha}=(l-p)^{d} \leq(l-p)^{p(\sigma-1)} .
$$

Let us consider the case $j>0$ and $n>0$. Let $a=m / j$ and $b=m /(m-j)$. Then, we have $1 / a+1 / b=1$. From the application of Young's inequality we have

$$
k^{j}(l-p)^{n(m-j) / m} \leq \frac{1}{a}\left(k^{j}\right)^{a}+\frac{1}{b}\left((l-p)^{n(m-j) / m}\right)^{b}=\frac{1}{a} k^{m}+\frac{1}{b}(l-p)^{n} \leq k^{m}+l^{n} .
$$

The result follows from $p(\sigma-1) \geq d$ and the fact that

$$
\frac{k^{j}(l-p)^{\alpha}}{k^{m}+l^{n}}=\frac{k^{j}(l-p)^{n(m-j) / m}}{k^{m}+l^{n}}(l-p)^{\alpha-n(m-j) / m} \leq(l-p)^{\alpha-n(m-j) / m}=(l-p)^{d} .
$$

As a direct consequence of Lemma 5, we have

Lemma 6. Let $m_{i}, m_{i+1}, n_{i}, n_{i+1}, p \in \mathbb{N}$ with $m_{i} \geq 1$ and $p \geq 1$. Suppose that $m_{i+1} \leq j<m_{i}$, $n_{i+1} \geq n_{i}$ and $\alpha>n_{i}+\left(n_{i+1}-n_{i}\right)\left(m_{i}-j\right) /\left(m_{i}-m_{i+1}\right)$ hold. Set $d=\alpha-n_{i}-\left(n_{i+1}-n_{i}\right)\left(m_{i}-\right.$ $j) /\left(m_{i}-m_{i+1}\right)$. Then, if $\sigma \geq(p+d) / p$ holds we have

$$
\frac{k^{j}(l-p)^{\alpha}}{k^{m_{i}} l^{n_{i}}+k^{m_{i+1}} l^{n_{i+1}}} \leq\left(\frac{l !}{(l-p) !}\right)^{\sigma-1}, \quad(k, l) \in \mathbb{N}^{*} \times \mathbb{N} \text { with } l \geq p .
$$

Proof. We apply Lemma 5 to

$$
\frac{k^{j}(l-p)^{\alpha}}{k^{m_{i}} l^{n_{i}}+k^{m_{i+1}} l^{n_{i+1}}} \leq \frac{k^{j-m_{i+1}}(l-p)^{\alpha-n_{i}}}{k^{m_{i}-m_{i+1}}+l^{n_{i+1}-n_{i}}} .
$$




\subsection{On an auxiliary equation}

In this subsection, we consider the auxiliary equation

$$
C\left(x ; k, x \partial_{x}\right) w=g(x) \in \mathbb{C}[[x]]
$$

under the assumption $\sigma_{0}>1$, where $C(x ; \lambda, \rho)$ is defined in (3). We note that the condition $\sigma_{0}>1$ is equivalent to the condition $\Lambda \backslash \mathcal{N}_{0} \neq \emptyset$ (or $\Lambda_{1} \backslash \mathcal{N}_{0} \neq \emptyset$ ). We set $\Lambda_{\text {out }}=\Lambda \backslash \mathcal{N}_{0}$. If $\Lambda_{\text {out }} \neq \emptyset$ holds, the irregularity $\sigma_{0}$ is defined by

$$
\sigma_{0}=\max _{(j, \alpha) \in \Lambda_{\text {out }}} \frac{p_{j, \alpha}+d_{j, \alpha}}{p_{j, \alpha}} .
$$

The definition of $p_{j, \alpha}$ described at the beginning of Section 2.3 allow us to express the coefficients $c_{j, \alpha}(x)$ as follows:

$$
c_{j, \alpha}(x)= \begin{cases}x^{p_{j, \alpha}} \gamma_{j, \alpha}(x), & \text { if }(j, \alpha) \in \Lambda_{1}, \\ c_{j, \alpha}(0)+x^{p_{j, \alpha}} \gamma_{j, \alpha}(x), & \text { if }(j, \alpha) \in \Lambda_{0} \backslash\{(m, 0)\},\end{cases}
$$

where $\gamma_{j, \alpha}(x) \in \mathbb{C}\{x\}$.

Observe that, in case $(j, \alpha) \in \Lambda_{1}$, the elements $p_{j, \alpha}$ are those in (8).

Proposition 3. Suppose the conditions $(N),(G P)$ are taken for granted, and $\sigma_{0}>1$. Then, for any $k \in \mathbb{N}^{*}$ and $g(x) \in \mathbb{C}[[x]]$ the equation (15) has a unique solution $w(x) \in \mathbb{C}[[x]]$, and it holds that

$$
\mathcal{B}_{\sigma}^{(m)}[|w|](x) \ll \frac{A(x)}{k^{m}} \mathcal{B}_{\sigma}^{(m)}[|g|](x)
$$

for any $\sigma \geq \sigma_{0}$, where

$$
A(x)=\frac{1}{c_{0}} \sum_{n \geq 0}\left(\frac{C_{1}}{c_{0}} \sum_{(j, \alpha) \in \Lambda} x^{p_{j, \alpha}} \mathcal{B}_{\sigma}^{(m)}\left[\left|\gamma_{j, \alpha}\right|\right](x)\right)^{n},
$$

$c_{0}>0$ is the constant in (7), and $C_{1}>0$ is a constant which is independent of $k$ and $g(x)$.

Proof. Take any $k \in \mathbb{N}^{*}$ and $g(x) \in \mathbb{C}[[x]]$. For the sake of simplicity, we adopt the following notation: $[\rho]_{0}=1 ;[\rho]_{\alpha}=\rho(\rho-1) \cdots(\rho-\alpha+1)$ (for $\left.\alpha \geq 1\right)$. Then, equation (15) is written in the form

$$
L\left(k, x \partial_{x}\right) w=g(x)+\sum_{(j, \alpha) \in \Lambda} x^{p_{j, \alpha}} \gamma_{j, \alpha}(x) k^{j}\left[x \partial_{x}\right]_{\alpha} w
$$

We set

$$
w(x)=\sum_{l \geq 0} w_{l} x^{l}, \quad g(x)=\sum_{l \geq 0} g_{l} x^{l}, \quad \gamma_{j, \alpha}(x)=\sum_{i \geq 0} \gamma_{j, \alpha, i} x^{i} .
$$

Then, by substituting these series into (19) and comparing the coefficients of $x^{l}$ at both sides of the equation, this is decomposed into the following recurrence formulas:

$$
L(k, l) w_{l}=g_{l}+\sum_{(j, \alpha) \in \Lambda} \sum_{i=0}^{l-p_{j, \alpha}} \gamma_{j, \alpha, i} k^{j}\left[l-p_{j, \alpha}-i\right]_{\alpha} w_{l-p_{j, \alpha}-i}, \quad l \in \mathbb{N} .
$$


Since $L(k, l) \neq 0$ for all $(k, l) \in \mathbb{N}^{*} \times \mathbb{N}, w_{l}$ is determined inductively for $l=0,1,2, \ldots$ Hence, equation (19) has a unique formal solution $w(x) \in \mathbb{C}[[x]]$.

Let us show (17). By the assumptions (N), (GP), Lemma 1 and Proposition 2 we have

$$
\begin{aligned}
\left|w_{l}\right| & \leq \frac{1}{L(k, l)}\left(\left|g_{l}\right|+\sum_{(j, \alpha) \in \Lambda} \sum_{i=0}^{l-p_{j, \alpha}}\left|\gamma_{j, \alpha, i}\right| k^{j}\left[l-p_{j, \alpha}-i\right]_{\alpha}\left|w_{l-p_{j, \alpha}-i}\right|\right) \\
& \leq \frac{1}{c_{0} \phi(k, l)}\left(\left|g_{l}\right|+\sum_{(j, \alpha) \in \Lambda} \sum_{i=0}^{l-p_{j, \alpha}}\left|\gamma_{j, \alpha, i}\right| k^{j}\left(l-p_{j, \alpha}\right)^{\alpha}\left|w_{l-p_{j, \alpha}-i}\right|\right) \\
& \leq \frac{1}{c_{0} k^{m}}\left|g_{l}\right|+\frac{1}{c_{0}} \sum_{(j, \alpha) \in \Lambda} \sum_{i=0}^{l-p_{j, \alpha}}\left|\gamma_{j, \alpha, i}\right| \frac{l !^{\sigma-1}}{\left(l-p_{j, \alpha}\right) ! !^{\sigma-1}}\left|w_{l-p_{j, \alpha}-i}\right| \\
& \leq \frac{1}{c_{0} k^{m}}\left|g_{l}\right|+\frac{C_{1}}{c_{0}} \sum_{(j, \alpha) \in \Lambda} \sum_{i=0}^{l-p_{j, \alpha}}\left|\gamma_{j, \alpha, i}\right| \frac{[l-m]_{+} !^{\sigma-1}}{\left[l-p_{j, \alpha}-m\right]_{+} !^{\sigma-1}}\left|w_{l-p_{j, \alpha}-i}\right|
\end{aligned}
$$

for some constant $C_{1}>0$. Taking into account that

$$
[i-m]_{+} !^{\sigma-1}\left[l-p_{j, \alpha}-i-m\right]_{+} !^{\sigma-1} \leq\left[l-p_{j, \alpha}-m\right]_{+} !^{\sigma-1}
$$

we conclude

$$
\mathcal{B}_{\sigma}^{(m)}[|w|] \ll \frac{1}{c_{0} k^{m}} \mathcal{B}_{\sigma}^{(m)}[|g|]+\frac{C_{1}}{c_{0}} \sum_{(j, \alpha) \in \Lambda} x^{p_{j, \alpha}} \mathcal{B}_{\sigma}^{(m)}\left[\left|\gamma_{j, \alpha}\right|\right] \times \mathcal{B}_{\sigma}^{(m)}[|w|],
$$

which yields (17) by setting $A(x)$ as in (18).

\section{Proof of (ii) of Theorem 2}

In this section, we give a proof of (ii) of Theorem 2 in the case $\sigma=\sigma_{0}$ and $s \geq s_{0}$, where $s_{0}$ is determined in (9). The first lemma provides a reformulation of the index $s_{0}$, which leans on the following construction.

For $\mu \in \mathbb{N}$ we define

$$
J_{\mu}=\left\{(i, \boldsymbol{\nu}) \in \mathbb{N} \times \mathbb{N}^{N} ; i+|\boldsymbol{\nu}| \geq 2,|\boldsymbol{\nu}| \geq 1,\left(\partial_{x}^{\mu} a_{i, \boldsymbol{\nu}}\right)(0) \neq 0\right\} .
$$

For $\mu \in \mathbb{N}$ and $\boldsymbol{\nu}=\left\{\nu_{j, \alpha}\right\}_{(j, \alpha) \in I_{m}}$ satisfying $|\boldsymbol{\nu}| \geq 1$ we set

$$
K_{\boldsymbol{\nu}}=\left\{(j, \alpha) \in I_{m} ; \nu_{j, \alpha}>0\right\}, \quad m_{\boldsymbol{\nu}, \mu}=\max _{(j, \alpha) \in K_{\boldsymbol{\nu}}}\left(j+\max \left\{\alpha, \mu+\sigma_{0}(\alpha-\mu)\right\}\right) .
$$

If $\mu \geq m$ we have $m_{\boldsymbol{\nu}, \mu} \leq m$ for any $\boldsymbol{\nu}$ with $|\boldsymbol{\nu}| \geq 1$. We have

Lemma 7. The index $s_{0}$ in (9) can be expressed in the form

$$
s_{0}=1+\max \left[0, \max _{0 \leq \mu<m}\left(\sup _{(i, \boldsymbol{\nu}) \in J_{\mu}} \frac{m_{\boldsymbol{\nu}, \mu}-m}{i+|\boldsymbol{\nu}|-1}\right)\right] .
$$


Proof. Set $f(\mu, j, \alpha)=j+\mu+\sigma_{0}(\alpha-\mu)-m$ : then $s_{0}$ is given by (9) in the form

$$
s_{0}=1+\max \left[0, \max _{0 \leq \mu<m}\left(\max _{(j, \alpha) \in I_{m}} \frac{f(\mu, j, \alpha)}{L_{\mu, j, \alpha}}\right)\right] .
$$

Therefore, $s_{0}$ is determined only by $(\mu, j, \alpha)$ satisfying $f(\mu, j, \alpha)>0$. Since $\left(\partial_{z_{j, \alpha}} \partial_{x}{ }^{\mu} R_{2}\right)(t, 0, \boldsymbol{z})$ is expressed in the form

$$
\left(\partial_{z_{j, \alpha}} \partial_{x}^{\mu} R_{2}\right)(t, 0, \boldsymbol{z})=\sum_{(i, \boldsymbol{\nu}) \in J_{\mu}, \nu_{j, \alpha}>0} \nu_{j, \alpha}\left(\partial_{x}^{\mu} a_{i, \boldsymbol{\nu}}\right)(0) t^{i} \boldsymbol{z}^{\boldsymbol{\nu}-e_{j, \alpha}}
$$

(where $e_{j, \alpha} \in \mathbb{N}^{N}$ is an $N$-vector defined by $\left\{\nu_{i, \beta}\right\}_{(i, \beta) \in I_{m}}$ with $\nu_{j, \alpha}=1$ and $\nu_{i, \beta}=0$ for $(i, \beta) \neq(j, \alpha))$, by the definition of $L_{\mu, j, \alpha}$ we have

$$
\begin{aligned}
s_{0} & =1+\max \left[0, \max _{0 \leq \mu<m}\left(\max _{(j, \alpha) \in I_{m}}\left(\sup _{(i, \boldsymbol{\nu}) \in J_{\mu}, \nu_{j, \alpha}>0} \frac{f(\mu, j, \alpha)}{i+|\boldsymbol{\nu}|-1}\right)\right)\right] \\
& =1+\max \left[0, \max _{0 \leq \mu<m}\left(\sup _{(i, \boldsymbol{\nu}) \in J_{\mu}}\left(\max _{(j, \alpha) \in K_{\boldsymbol{\nu}}} \frac{f(\mu, j, \alpha)}{i+|\boldsymbol{\nu}|-1}\right)\right)\right] .
\end{aligned}
$$

We set $g(\mu, j, \alpha)=j+\max \left\{\alpha, \mu+\sigma_{0}(\alpha-\mu)\right\}-m$. If $\alpha \leq \mu$ we have $f(\mu, j, \alpha) \leq 0$ and $g(\mu, j, \alpha) \leq 0$. If $\alpha>\mu$ we have $g(\mu, j, \alpha)=f(\mu, j, \alpha)$. Therefore, $s_{0}$ is determined only by $(\mu, j, \alpha)$ with $\alpha>\mu$ and

$$
s_{0}=1+\max \left[0, \max _{0 \leq \mu<m}\left(\sup _{(i, \boldsymbol{\nu}) \in J_{\mu}}\left(\max _{(j, \alpha) \in K_{\nu}} \frac{g(\mu, j, \alpha)}{i+|\boldsymbol{\nu}|-1}\right)\right)\right] .
$$

This proves (20).

Suppose the conditions (N), (GP) and $\sigma_{0}>1$ hold. Then, we have $\Lambda_{\text {out }} \neq \emptyset$. Let

$$
u(t, x)=\sum_{k \geq 1} u_{k}(x) t^{k} \in(\mathbb{C}[[x]])[[t]]
$$

be the unique formal solution of (1). Then, $u_{k}(x)(k=1,2, \ldots)$ are determined as the solutions of the recurrence formulas:

$$
C\left(x ; k, x \partial_{x}\right) u_{k}=f_{k}(x), \quad k=1,2, \ldots
$$

with $f_{1}(x)=a(x)$ and for $k \geq 2$

$$
f_{k}(x)=\sum_{2 \leq i+|\boldsymbol{\nu}| \leq k} a_{i, \boldsymbol{\nu}}(x) \sum_{i+|k(\boldsymbol{\nu})|=k} \prod_{(j, \alpha) \in I_{m}} \prod_{h=1}^{\nu_{j, \alpha}}\left(k_{j, \alpha}(h)\right)^{j} \partial_{x}^{\alpha} u_{k_{j, \alpha}(h)},
$$

where $\boldsymbol{\nu}=\left\{\nu_{j, \alpha}\right\}_{(j, \alpha) \in I_{m}}$ and $|k(\boldsymbol{\nu})|=\sum_{(j, \alpha) \in I_{m}}\left(k_{j, \alpha}(1)+\cdots+k_{j, \alpha}\left(\nu_{j, \alpha}\right)\right)$. By Proposition 3 we have

$$
\mathcal{B}_{\sigma_{0}}^{(m)}\left[\left|u_{k}\right|\right](x) \ll \frac{A(x)}{k^{m}} \mathcal{B}_{\sigma_{0}}^{(m)}\left[\left|f_{k}\right|\right](x), \quad k=1,2, \ldots
$$

Since $f_{1}(x)$ is holomorphic at $x=0$, by $(21)$ we see that $\mathcal{B}_{\sigma_{0}}^{(m)}\left[\left|u_{1}\right|\right](x)$ is holomorphic at $x=0$. We can show by induction on $k$ that $\mathcal{B}_{\sigma_{0}}^{(m)}\left[\left|u_{k}\right|\right](x)(k \geq 1)$ are all holomorphic at $x=0$. Thus, we have that $u_{k}(x) \in G\{x\}_{\sigma_{0}}$ for all $k \geq 1$. 


\subsection{On a majorant equation}

Let $0<R \leq 1$ be small enough so that $A(x) \in \mathcal{O}\left(\bar{D}_{R}\right), a_{i, \boldsymbol{\nu}}(x) \in \mathcal{O}\left(\bar{D}_{R}\right)(i+|\boldsymbol{\nu}| \geq 2)$ and $\mathcal{B}_{\sigma}^{(m)}\left[\left|u_{1}\right|\right](x) \in \mathcal{O}\left(\bar{D}_{R}\right)$. We take $A>0$ so that

$$
\mathcal{B}_{\sigma_{0}}^{(m-\mu)}\left[\partial_{x}^{\alpha}\left|u_{1}\right|\right](x) \ll \frac{A}{R-x}, \quad 0 \leq \mu \leq m,(j, \alpha) \in I_{m},
$$

and $A_{i, \boldsymbol{\nu}} \geq 0(i+|\boldsymbol{\nu}| \geq 2)$ such that

$$
A(x) \mathcal{B}_{\sigma_{0}}^{(m)}\left[\left|a_{i, \boldsymbol{\nu}}\right|\right](x) \ll \frac{A_{i, \boldsymbol{\nu}}}{R-x} \text { and } \sum_{i+|\boldsymbol{\nu}| \geq 2} A_{i, \boldsymbol{\nu}} t^{i} Y^{|\boldsymbol{\nu}|} \in \mathbb{C}\{t, Y\} .
$$

We take $L \in \mathbb{N}^{*}$ so that $L \geq m \sigma_{0}$. Then we have $L \geq j+\max \left\{\alpha, \mu+\sigma_{0}(\alpha-\mu)\right\}$ for any $0 \leq \mu<m$ and $(j, \alpha) \in I_{m}$. Set $H=\left(3 m e \sigma_{0}\right)^{m \sigma_{0}}$. Under these notations, let us consider the functional equation

$$
Y=\frac{A}{(R-x)^{m \sigma_{0}}} t+\frac{1}{(R-x)^{m \sigma_{0}}} \sum_{i+|\boldsymbol{\nu}| \geq 2} \frac{A_{i, \boldsymbol{\nu}}(i+|\boldsymbol{\nu}|)^{L}}{(R-x)^{m \sigma_{0}(3 i+2|\boldsymbol{\nu}|-3)}} t^{i}(H Y)^{|\boldsymbol{\nu}|}
$$

with respect to $(t, Y)$, where $x \in D_{R}$ is regarded as a parameter. By the implicit function theorem we see that for any $x \in D_{R}$ the equation (24) has a unique holomorphic solution $Y=Y(t)$ in a neighborhood of $t=0$ satisfying $Y(0)=0$. The coefficients of the Taylor expansion $Y=\sum_{k \geq 1} Y_{k} t^{k}$, are determined by the following recurrence formulas:

$$
Y_{1}=\frac{A}{(R-x)^{m \sigma_{0}}},
$$

and for $k \geq 2$

$$
Y_{k}=\frac{1}{(R-x)^{m \sigma_{0}}} \sum_{2 \leq i+|\boldsymbol{\nu}| \leq k} \frac{A_{i, \boldsymbol{\nu}}(i+|\boldsymbol{\nu}|)^{L}}{(R-x)^{m \sigma_{0}(3 i+2|\boldsymbol{\nu}|-3)}}\left[\sum_{i+|k(\boldsymbol{\nu})|=k} \prod_{(j, \alpha) \in I_{m}} \prod_{h=1}^{\nu_{j, \alpha}} H Y_{k_{j, \alpha}}(h)\right] .
$$

Moreover, by induction on $k$ we can show that $Y_{k}$ has the form

$$
Y_{k}=\frac{C_{k}}{(R-x)^{m \sigma_{0}(3 k-2)}}, \quad k=1,2, \ldots
$$

where $C_{1}=A$ and $C_{k} \geq 0(k \geq 2)$ are constants which are independent of the parameter $x$.

Lemma 8. Assume that $s \geq s_{0}$. Then, for any $k=1,2, \ldots$ we have

$$
\mathcal{B}_{\sigma_{0}}^{(m-\mu)}\left[k^{j} \partial_{x}^{\alpha}\left|u_{k}\right|\right](x) \ll \frac{(k-1) !^{s-1}}{k^{L-j-\max \left\{\alpha, \mu+\sigma_{0}(\alpha-\mu)\right\}}} H Y_{k}
$$

for any $0 \leq \mu \leq m$ and $(j, \alpha) \in I_{m}$.

\subsection{Proof of Lemma 8}

Proof. In the case $k=1$, by (22) and (25) we have

$$
\mathcal{B}_{\sigma_{0}}^{(m-\mu)}\left[1^{j} \partial_{x}^{\alpha}\left|u_{1}\right|\right](x) \ll \frac{A}{R-x} \ll \frac{A}{(R-x)^{m \sigma_{0}}}=Y_{1} \ll H Y_{1}
$$


for any $0 \leq \mu \leq m$ and $(j, \alpha) \in I_{m}$. Hence we have $(27)_{k}$ for $k=1$. Let us show the general case by induction on $k$.

Let $k \geq 2$, and suppose that the equation is already proved for all $1 \leq p<k$. We express

$$
a_{i, \boldsymbol{\nu}}(x)=a_{i, \boldsymbol{\nu}, 0}+a_{i, \boldsymbol{\nu}, 1} x+\cdots+a_{i, \boldsymbol{\nu}, m-1} x^{m-1}+x^{m} a_{i, \boldsymbol{\nu}, m}(x) .
$$

Then,

$$
f_{k}(x)=\sum_{\mu=0}^{m} x^{\mu} \sum_{2 \leq i+|\boldsymbol{\nu}| \leq k} a_{i, \boldsymbol{\nu}, \mu} \sum_{i+|k(\boldsymbol{\nu})|=k} \prod_{(j, \alpha) \in I_{m}} \prod_{h=1}^{\nu_{j, \alpha}}\left(k_{j, \alpha}(h)\right)^{j} \partial_{x}^{\alpha} u_{k_{j, \alpha}(h)}
$$

and so by Lemma 3 , and setting $\mathcal{A}_{i, \boldsymbol{\nu}, \mu}=\left|a_{i, \boldsymbol{\nu}, \mu}\right|$ for $0 \leq \mu \leq m-1$, and $\mathcal{A}_{i, \boldsymbol{\nu}, m}=\mathcal{B}_{\sigma_{0}}\left[\left|a_{i, \boldsymbol{\nu}, m}\right|\right]$ we have

$$
\mathcal{B}_{\sigma_{0}}^{(m)}\left[\left|f_{k}\right|\right] \ll \sum_{\mu=0}^{m} x^{\mu} \sum_{2 \leq i+|\boldsymbol{\nu}| \leq k} \mathcal{A}_{i, \boldsymbol{\nu}, \mu}\left[\sum_{i+|k(\boldsymbol{\nu})|=k} \prod_{(j, \alpha) \in I_{m}} \prod_{h=1}^{\boldsymbol{\nu}_{j, \alpha}} \mathcal{B}_{\sigma_{0}}^{(m-\mu)}\left[\left(k_{j, \alpha}(h)\right)^{j} \partial_{x}^{\alpha} u_{k_{j, \alpha}(h)}\right]\right] .
$$

Thus, by (21), the definition of $m_{\boldsymbol{\nu}, \mu}$ and the induction hypothesis we have

$$
\mathcal{B}_{\sigma_{0}}^{(m)}\left[\left|u_{k}\right|\right] \ll \frac{A(x)}{k^{m}} \sum_{\mu=0}^{m} x^{\mu} \sum_{2 \leq i+|\boldsymbol{\nu}| \leq k} \mathcal{A}_{i, \boldsymbol{\nu}, \mu}\left[\sum_{i+|k(\boldsymbol{\nu})|=k} \prod_{(j, \alpha) \in I_{m}} \prod_{h=1}^{\nu_{j, \alpha}} \frac{\left(k_{j, \alpha}(h)-1\right) !^{s-1}}{\left(k_{j, \alpha}(h)\right)^{L-m_{\boldsymbol{\nu}, \mu}}} H Y_{k_{j, \alpha}}(h)\right] .
$$

Observe the condition $L-m_{\boldsymbol{\nu}, \mu} \geq 0$ follows from the choice of $L$ so that $L \geq \sigma_{0} m$.

Lemma 9. Under the above situation, $|\boldsymbol{\nu}| \geq 1$ and $\mathcal{A}_{i, \boldsymbol{\nu}, \mu} \neq 0\left(\right.$ or $\left.\mathcal{A}_{i, \boldsymbol{\nu}, m}(x) \not \equiv 0\right)$ we have

$$
\begin{aligned}
& \frac{(k-i-|\boldsymbol{\nu}|) !^{s-1}}{k^{L+m-m_{\boldsymbol{\nu}, \mu}}} \leq \frac{(k-1) !^{s-1}(i+|\boldsymbol{\nu}|)^{\left[m_{\boldsymbol{\nu}, \mu}-m\right]_{+}}}{k^{L}}, \\
& \frac{1}{k^{m}} \prod_{(j, \alpha) \in I_{m}} \prod_{h=1}^{\nu_{j, \alpha}} \frac{\left(k_{j, \alpha}(h)-1\right) !^{!^{-1}}}{\left(k_{j, \alpha}(h)\right)^{L-m_{\boldsymbol{\nu}, \mu}}} \leq \frac{(k-1) !^{s-1}(i+|\boldsymbol{\nu}|)^{L}}{k^{L}} .
\end{aligned}
$$

Proof. Let us show (29). If $m \geq m_{\boldsymbol{\nu}, \mu}$ we have $\left[m_{\boldsymbol{\nu}, \mu}-m\right]_{+}=0$ and so

$$
\frac{(k-i-|\boldsymbol{\nu}|) !^{s-1}}{k^{L+m-m_{\boldsymbol{\nu}, \mu}}} \leq \frac{(k-1) !^{s-1}}{k^{L}}=\frac{(k-1) !^{s-1}(i+|\boldsymbol{\nu}|)^{\left[m_{\boldsymbol{\nu}, \mu}-m\right]_{+}}}{k^{L}} .
$$

If $m_{\boldsymbol{\nu}, \mu}>m$, by the assumption $s \geq s_{0}$ and Lemma 7 we have $(i+|\boldsymbol{\nu}|-1)(s-1) \geq m_{\boldsymbol{\nu}, \mu}-m$ and so we have

$$
\begin{aligned}
\frac{(k-i-|\boldsymbol{\nu}|) !^{s-1}}{k^{L+m-m_{\boldsymbol{\nu}, \mu}}} & \leq \frac{(k-1) !^{s-1}}{k^{L}} \frac{k^{m_{\boldsymbol{\nu}, \mu}-m}}{(k-i-|\boldsymbol{\nu}|+1)^{m_{\boldsymbol{\nu}, \mu}-m}} \\
& \leq \frac{(k-1) !^{s-1}}{k^{L}}\left(1+\frac{i+|\boldsymbol{\nu}|-1}{k-i-|\boldsymbol{\nu}|+1}\right)^{m_{\boldsymbol{\nu}, \mu}-m} \leq \frac{(k-1) !^{s-1}}{k^{L}}(i+|\boldsymbol{\nu}|)^{m_{\boldsymbol{\nu}, \mu}-m} .
\end{aligned}
$$

This proves (29). In order to prove (30), we note that, if $k_{j} \geq 1(j=1, \ldots,|\boldsymbol{\nu}|)$ and $k_{1}+\cdots+$ $k_{|\boldsymbol{\nu}|}=k-i$ hold, then we have $k_{j} \leq\left(k_{1} \cdots k_{|\boldsymbol{\nu}|}\right)$ for $j=1, \ldots,|\boldsymbol{\nu}|$ and so $k-i=k_{1}+\cdots+k_{|\boldsymbol{\nu}|} \leq$ $|\boldsymbol{\nu}|\left(k_{1} \cdots k_{|\boldsymbol{\nu}|}\right)$ which yields $k \leq(i+|\boldsymbol{\nu}|)\left(k_{1} \cdots k_{|\boldsymbol{\nu}|}\right)$. Therefore, by the same argument we have

$$
\prod_{(j, \alpha) \in I_{m}} \prod_{h=1}^{\nu_{j, \alpha}} \frac{1}{\left(k_{j, \alpha}(h)\right)^{L-m_{\boldsymbol{\nu}, \mu}}} \leq\left(\frac{i+|\boldsymbol{\nu}|}{k}\right)^{L-m_{\boldsymbol{\nu}, \mu}} .
$$


Hence, by the condition $i+|k(\boldsymbol{\nu})|=k$ and (29) we have

$$
\begin{aligned}
& \frac{1}{k^{m}} \prod_{(j, \alpha) \in I_{m}} \prod_{h=1}^{\nu_{j, \alpha}} \frac{\left(k_{j, \alpha}(h)-1\right) !^{s-1}}{\left(k_{j, \alpha}(h)\right)^{L-m_{\boldsymbol{\nu}, \mu}}} \leq \frac{(|k(\boldsymbol{\nu})|-|\boldsymbol{\nu}|) !^{s-1}}{k^{m}} \prod_{(j, \alpha) \in I_{m}} \prod_{h=1}^{\nu_{j, \alpha}} \frac{1}{\left(k_{j, \alpha}(h)\right)^{L-m_{\boldsymbol{\nu}, \mu}}} \\
& \quad \leq \frac{(k-i-|\boldsymbol{\nu}|) !^{s-1}}{k^{m}} \times \frac{(i+|\boldsymbol{\nu}|)^{L-m_{\boldsymbol{\nu}, \mu}}}{k^{L-m_{\boldsymbol{\nu}, \mu}}} \leq \frac{(k-1) !^{s-1}}{k^{L}}(i+|\boldsymbol{\nu}|)^{\left[m_{\boldsymbol{\nu}, \mu}-m\right]_{+}} \times(i+|\boldsymbol{\nu}|)^{L-m_{\boldsymbol{\nu}, \mu}}
\end{aligned}
$$

which proves (30).

$$
\mathcal{B}_{\sigma_{0}}^{(m)}\left[\left|u_{k}\right|\right] \ll \frac{(k-1) !^{!^{-1}}}{k^{L}} A(x) \sum_{\mu=0}^{m} x^{\mu} \sum_{2 \leq i+|\boldsymbol{\nu}| \leq k} \mathcal{A}_{i, \boldsymbol{\nu}, \mu}(i+|\boldsymbol{\nu}|)^{L}\left[\sum_{i+|k(\boldsymbol{\nu})|=k} \prod_{(j, \alpha) \in I_{m}} \prod_{h=1}^{\nu_{j, \alpha}} H Y_{k_{j, \alpha}(h)}\right] .
$$

By the definition of $\mathcal{A}_{i, \boldsymbol{\nu}, \mu}(0 \leq \mu \leq m)$ we have $\sum_{\mu=0}^{m} x^{\mu} \mathcal{A}_{i, \boldsymbol{\nu}, \mu}=\mathcal{B}_{\sigma_{0}}^{(m)}\left[\left|a_{i, \boldsymbol{\nu}}\right|\right](x)$, and by (23) we have

$$
A(x) \sum_{\mu=0}^{m} x^{\mu} \mathcal{A}_{i, \boldsymbol{\nu}, \mu}=A(x) \mathcal{B}_{\sigma_{0}}^{(m)}\left[\left|a_{i, \boldsymbol{\nu}}\right|\right](x) \ll \frac{A_{i, \boldsymbol{\nu}}}{R-x} .
$$

By applying this to (31), and by (26) we derive

$$
\mathcal{B}_{\sigma_{0}}^{(m)}\left[\left|u_{k}\right|\right] \ll \frac{(k-1) !^{s-1}}{k^{L}}(R-x)^{m \sigma_{0}} Y_{k}=\frac{(k-1) !^{s-1}}{k^{L}} \frac{C_{k}}{(R-x)^{m \sigma_{0}(3 k-3)}} .
$$

If $\alpha \leq \mu$, by Lemma 3, (32) and Corollary 2 we have

$$
\begin{aligned}
\mathcal{B}_{\sigma_{0}}^{(m-\mu)}\left[k^{j} \partial_{x}^{\alpha}\left|u_{k}\right|\right] & \ll k^{j} \mathcal{B}_{\sigma_{0}}^{(m-\alpha)}\left[\partial_{x}^{\alpha}\left|u_{k}\right|\right] \ll \frac{(k-1) !^{!-1}}{k^{L-j}} \frac{\prod_{i=0}^{\alpha-1}\left(m \sigma_{0}(3 k-3)+i\right) \times C_{k}}{(R-x)^{m \sigma_{0}(3 k-3)+\alpha}} \\
& \ll \frac{(k-1) !^{s-1}}{k^{L-j-\alpha}} \frac{\left(3 m \sigma_{0}\right)^{\alpha} C_{k}}{(R-x)^{m \sigma_{0}(3 k-2)}} \ll \frac{(k-1) !^{s-1}}{k^{L-j-\alpha}} H Y_{k}(x) .
\end{aligned}
$$

If $\mu<\alpha$, the application of (32) and Corollary 2 yield

$$
\mathcal{B}_{\sigma_{0}}^{(m-\mu)}\left[k^{j} \partial_{x}^{\alpha}\left|u_{k}\right|\right]=k^{j} \mathcal{B}_{\sigma_{0}}^{(m-\mu)}\left[\partial_{x}^{(\alpha-\mu)+\mu}\left|u_{k}\right|\right] \ll \frac{(k-1) !^{s-1}}{k^{L-j}} \frac{A(\mu, \alpha) C_{k}}{(R-x)^{m \sigma_{0}(3 k-3)+\mu+\sigma_{0}(\alpha-\mu)}}
$$

where

$$
A(\mu, \alpha)=\prod_{i=0}^{\mu-1}\left(m \sigma_{0}(3 k-3)+i\right) \prod_{h=1}^{\alpha-\mu}\left[\left(m \sigma_{0}(3 k-3)+\mu+h \sigma_{0}\right)^{\sigma_{0}} e^{\sigma_{0}}\right] .
$$

Since $A(\mu, \alpha) \leq k^{\mu+\sigma_{0}(\alpha-\mu)}\left(3 m \sigma_{0}\right)^{\sigma_{0} \alpha} e^{\sigma_{0}(\alpha-\mu)}$, we have

$$
\begin{aligned}
\mathcal{B}_{\sigma_{0}}^{(m-\mu)}\left[k^{j} \partial_{x}^{\alpha}\left|u_{k}\right|\right] & \ll \frac{(k-1) !^{s-1}}{k^{L-j-\left(\mu+\sigma_{0}(\alpha-\mu)\right)}} \frac{\left(3 m \sigma_{0}\right)^{\sigma_{0} \alpha} e^{\sigma_{0}(\alpha-\mu)} C_{k}}{(R-x)^{m \sigma_{0}(3 k-3)+\mu+\sigma_{0}(\alpha-\mu)}} \\
& \ll \frac{(k-1) !^{s-1}}{k^{L-j-\left(\mu+\sigma_{0}(\alpha-\mu)\right)}} \frac{H C_{k}}{(R-x)^{m \sigma_{0}(3 k-3)+m \sigma_{0}}}=\frac{(k-1) !^{s-1}}{k^{L-j-\left(\mu+\sigma_{0}(\alpha-\mu)\right)}} H Y_{k} .
\end{aligned}
$$

By (33) and (34) we have (30). This proves Lemma 8. 


\subsection{Completion of the proof of (ii) of Theorem 2}

By Lemma 8 we have

$$
\sum_{k \geq 1} \frac{\mathcal{B}_{\sigma_{0}}\left[\left|u_{k}\right|\right](x)}{(k-1) !^{s-1}} t^{k} \ll \sum_{k \geq 1} \frac{\mathcal{B}_{\sigma_{0}}^{(m)}\left[\left|u_{k}\right|\right](x)}{(k-1) !^{s-1}} t^{k} \ll \sum_{k \geq 1} \frac{1}{k^{L}} H Y_{k}(x) t^{k}
$$

Take any $r \in(0, R)$. We know there is $\delta>0$ such that $\sum_{k \geq 1} Y_{k}(r) t^{k}$ is convergent for $|t| \leq \delta$. Then, for $|t| \leq \delta$ we have

$$
\sum_{k \geq 1} \mathcal{B}_{\sigma_{0}}\left[\left|u_{k}\right|\right](r) \frac{|t|^{k}}{(k-1) !^{s-1}} \leq H \sum_{k \geq 1} Y_{k}(r) \delta^{k}<\infty .
$$

This proves that $u(t, x) \in G\{t, x\}_{\left(s, \sigma_{0}\right)}$ holds, and we have (ii) of Theorem 2 .

\section{$5 \quad$ Proof of Theorem 3}

Suppose the conditions $\left.\left.\mathrm{A}_{1}\right) \sim \mathrm{A}_{3}\right), \mathcal{L} \neq \emptyset$, and $\left.\left.\mathrm{c}_{1}\right) \sim \mathrm{c}_{4}\right)$ hold. Since $L(\lambda, \rho)$ is defined by

$$
L(\lambda, \rho)=\lambda^{m}+\sum_{(j, \alpha) \in\left(\Lambda_{0} \backslash\{(m, 0)\}\right)}\left(-c_{j, \alpha}(0)\right) \lambda^{j}[\rho]_{\alpha}
$$

(where $[\rho]_{0}=1$ and $[\rho]_{\alpha}=\rho(\rho-1) \cdots(\rho-\alpha+1)$ for $\alpha \geq 1$ ) and since $-c_{j, \alpha}(0)>0$ holds for any $(j, \alpha) \in\left(\Lambda_{0} \backslash\{(m, 0)\}\right)$, we have $L(k, l) \geq k^{m} \geq 1$ for any $(k, l) \in \mathbb{N}^{*} \times \mathbb{N}$. This means that the condition $(\mathrm{N})$ is satisfied which entails that the equation (1) has a unique formal solution $u(t, x) \in \mathbb{C}[[t, x]]$ satisfying $u(0, x) \equiv 0$.

Since $\left(m_{i}, n_{i}\right) \in \Lambda_{0}$ for $i=1, \ldots, p$ and since the coefficients of $\lambda^{m_{i}}[\rho]_{n_{i}}(i=1, \ldots, p)$ in $L(\lambda, \rho)$ are all positive, we have $L(k, l) \geq c_{0} \phi(k, l)$ on $\left\{(k, l) \in \mathbb{N}^{*} \times \mathbb{N} ; l \geq m\right\}$ for some $c_{0}>0$. Since $m^{n_{i}} \geq l^{n_{i}}$ for $0 \leq l \leq m-1$, by setting $\delta_{i}=1 / m^{n_{i}}$ we have $L(k, l) \geq k^{m} \geq \delta_{i} k^{m_{i}} l^{n_{i}}$ on $\left\{(k, l) \in \mathbb{N}^{*} \times \mathbb{N} ; l<m\right\}$. Hence, we have $L(k, l) \geq c_{1} \phi(k, l)$ on $\left\{(k, l) \in \mathbb{N}^{*} \times \mathbb{N} ; l<m\right\}$ for some $c_{1}>0$. Thus, by Lemma 1 we see that the generalized Poincaré condition (GP) is satisfied. Hence, by Theorem 2 we have $u(t, x) \in G\{t, x\}_{(s, \sigma)}$ provided that $(s, \sigma)$ satisfies $s \geq s_{0}$ and $\sigma \geq \sigma_{0}$.

\subsection{Proof of the converse statement}

Let us show the converse statement. For $\mu \in\{0,1, \ldots, m-1\}$ and $(j, \alpha) \in I_{m}$, we define

$$
J_{\mu, j, \alpha}=\left\{(i, \nu) \in J_{\mu}:(j, \alpha) \in K_{\nu}\right\},
$$

where $J_{\mu}$ and $K_{\nu}$ are given in Section 4 . We set

$$
\mathcal{L}_{0}=\bigcup_{\mu=0}^{m-1} \bigcup_{(j, \alpha) \in I_{m}, \mu<\alpha} J_{\mu, j, \alpha} .
$$

We distinguish two cases: $\mathcal{L}_{0} \neq \emptyset$ and $\mathcal{L}_{0}=\emptyset$ (but $\mathcal{L} \neq \emptyset$ ).

First, assume that $\mathcal{L}_{0} \neq \emptyset$. In case it holds that $u(t, x) \in G\{t, x\}_{(s, \sigma)}$ for some $s \geq 1$ and $\sigma \geq 1$, then since $s_{0}$ is expressed in the form (20) and

$$
j+\max \left\{\alpha, \mu+\sigma_{0}(\alpha-\mu)\right\}-m= \begin{cases}j+\alpha+\left(\sigma_{0}-1\right)(\alpha-\mu)-m, & \text { if } \alpha>\mu, \\ j+\alpha-m \leq 0, & \text { if } \alpha \leq \mu,\end{cases}
$$


in order to show the conditions $s \geq s_{0}$ and $\sigma \geq \sigma_{0}$, it is enough to prove that the two conditions

$$
\sigma \geq \frac{p_{h, \beta}+d_{h, \beta}}{p_{h, \beta}}, \quad s-1 \geq \frac{j+\alpha+\left(d_{h, \beta} / p_{h, \beta}\right)(\alpha-\mu)-m}{i+|\nu|-1}
$$

hold for any $(h, \beta) \in \Lambda_{\text {out }}, \mu \in\{0,1, \ldots, m-1\},(i, \nu) \in J_{\mu}$ and $(j, \alpha) \in K_{\boldsymbol{\nu}}$ satisfying $\alpha>\mu$. The condition $\mathcal{L}_{0} \neq \emptyset$ implies that there exists at least one such 5 -tuple $(\mu, i, \nu, j, \alpha)$.

On the other hand, if $\mathcal{L}_{0}=\emptyset$, we have $s_{0}=1$ and so we only need to show condition (35) for any $(h, \beta) \in \Lambda_{\text {out }}$. Since $\mathcal{L} \neq \emptyset$ is assumed, there exists $(\mu, i, \nu)$ such that $\partial_{x}^{\mu} a_{i, \nu}(0) \neq 0$. For such indices, and bearing in mind that $\mathcal{L}_{0}=\emptyset$, we arrive at the property that $(j, \alpha) \in K_{\nu}$ implies that $\mu \geq \alpha$.

In both cases, we take $(h, \beta) \in \Lambda_{\text {out }}, \mu \in \mathbb{N},(i, \nu) \in J_{\mu}$ and $(j, \alpha) \in K_{\boldsymbol{\nu}}$. In case $\mathcal{L}_{0} \neq \emptyset$ we may suppose $\mu<\alpha$; but if $\mathcal{L}_{0}=\emptyset$, then only $\mu \geq \alpha$ may apply.

Note that equation (1) is written as

$$
L\left(t \partial_{t}, x \partial_{x}\right) u=a(x) t+\sum_{(j, \alpha) \in \Lambda} x^{p_{j, \alpha}} \gamma_{j, \alpha}(x)\left(t \partial_{t}\right)^{j}\left[x \partial_{x}\right]_{\alpha} u+\sum_{i+|\boldsymbol{\nu}| \geq 2} a_{i, \boldsymbol{\nu}}(x) t^{i} \prod_{(j, \alpha) \in I_{m}}\left[\left(t \partial_{t}\right)^{j} \partial_{x}^{\alpha} u\right]^{\nu_{j, \alpha}},
$$

and that its formal solution $u(t, x)=\sum_{k \geq 1} u_{k}(x) t^{k} \in \mathbb{C}[[t, x]]$ satisfies that $u(t, x) \gg 0$ and $L\left(1, x \partial_{x}\right) u_{1}(x)=a(x)$. Since $\partial_{x}^{l} u(t, x) \gg\left(\partial_{x}^{l} u_{1}\right)(0) t=\left(\partial_{x}^{l} a\right)(0) t / L(1, l)$ for any $l \in \mathbb{N}$, we have

$$
\begin{aligned}
L\left(t \partial_{t}, x \partial_{x}\right) u & \gg \frac{\left(\partial_{x}^{m} a\right)(0)}{m !} x^{m} t+\gamma_{h, \beta}(0) x^{p_{h, \beta}}\left(t \partial_{t}\right)^{h}\left[x \partial_{x}\right]_{\beta} u \\
& +\frac{\left(\partial_{x}^{\mu} a_{i, \boldsymbol{\nu}}\right)(0)}{\mu !} x^{\mu} t^{i+|\boldsymbol{\nu}|-1} \prod_{(k, \gamma) \neq(j, \alpha)}\left(\frac{\left(\partial^{\gamma} a\right)(0)}{L(1, \gamma)}\right)^{\nu_{k, \gamma}} \times\left(\frac{\left(\partial^{\alpha} a\right)(0)}{L(1, \alpha)}\right)^{\nu_{j, \alpha}-1} \times\left(t \partial_{t}\right)^{j} \partial_{x}^{\alpha} u
\end{aligned}
$$

Thus, by setting

$$
A=\frac{\left(\partial_{x}^{m} a\right)(0)}{m !}, \quad B=\gamma_{h, \beta}(0), \quad C=\frac{\left(\partial_{x}^{\mu} a_{i, \nu}\right)(0)}{\mu !} \prod_{(k, \gamma) \neq(j, \alpha)}\left(\frac{\left(\partial^{\gamma} a\right)(0)}{L(1, \gamma)}\right)^{\nu_{k, \gamma}} \times\left(\frac{\left(\partial^{\alpha} a\right)(0)}{L(1, \alpha)}\right)^{\nu_{j, \alpha}-1},
$$

we have $A>0, B>0, C>0$ and

$$
L\left(t \partial_{t}, x \partial_{x}\right) u \gg A x^{m} t+B x^{p_{h, \beta}}\left(t \partial_{t}\right)^{h}\left[x \partial_{x}\right]_{\beta} u+C x^{\mu} t^{i+|\nu|-1}\left(t \partial_{t}\right)^{j} \partial_{x}^{\alpha} u .
$$

Now, let us consider the equation

$$
L\left(t \partial_{t}, x \partial_{x}\right) w=A x^{m} t+B x^{p_{h, \beta}}\left(t \partial_{t}\right)^{h}\left[x \partial_{x}\right]_{\beta} w+C x^{\mu} t^{i+|\nu|-1}\left(t \partial_{t}\right)^{j} \partial_{x}^{\alpha} w .
$$

Lemma 10. Under the above situation, the equation (37) has a unique formal solution $w(t, x) \in$ $\mathbb{C}[[t, x]]$ satisfying $w(0, x) \equiv 0$. The following statements hold:

(1) If $\mu<\alpha$, then $w(t, x)$ belongs to the class $G\{t, x\}_{\left(s^{\prime}, \sigma^{\prime}\right)}$ if and only if $\left(s^{\prime}, \sigma^{\prime}\right)$ satisfies

$$
\sigma^{\prime} \geq \frac{p_{h, \beta}+d_{h, \beta}}{p_{h, \beta}}, \quad s^{\prime}-1 \geq \frac{j+\alpha+\left(d_{h, \beta} / p_{h, \beta}\right)(\alpha-\mu)-m}{i+|\nu|-1} .
$$

(2) If $\mu \geq \alpha$, then $w(t, x)$ belongs to the class $G\{t, x\}_{\left(s^{\prime}, \sigma^{\prime}\right)}$ if and only if $\left(s^{\prime}, \sigma^{\prime}\right)$ satisfies

$$
\sigma^{\prime} \geq \frac{p_{h, \beta}+d_{h, \beta}}{p_{h, \beta}}, \quad s^{\prime}-1 \geq 0 .
$$

The proof of this lemma will be given in Section 5.3.

By (36) and (37), it holds that $u(t, x) \gg w(t, x)$. Since $u(t, x) \in G\{t, x\}_{(s, \sigma)}$ is assumed, we have $w(t, x) \in G\{t, x\}_{(s, \sigma)}$, and so by Lemma 10 we have the conditions (35) in the case $\mu<\alpha$, and just the first condition in (35) for $\mu \geq \alpha$.

Thus, to complete the proof of Theorem 3 it is enough to show Lemma 10 above. 


\subsection{Some lemmas}

Before the proof of Lemma 10, let us give some lemmas which are needed in that proof. We note that by the assumption $\left.\mathrm{c}_{2}\right)$ we have $L(k, l) \geq k^{m} \geq 1$ for any $(k, l) \in \mathbb{N}^{*} \times \mathbb{N}$.

Lemma 11. The following statements hold:

(i) There is a constant $c_{1}>0$ such that $L(k, l) \leq c_{1} \phi(k, l)$ for every $(k, l) \in \mathbb{N}^{*} \times \mathbb{N}$.

(ii) Let $a>0$ and $q \in \mathbb{N}^{*}$. Then, there is $c_{2}>0$ with $L(k q+1, a) \leq c_{2}(k+1)^{m}$ for all $k \in \mathbb{N}^{*}$.

(iii) Let $1 \leq i \leq p$, and let $-s_{i}$ be the slope of $\Gamma_{i}$. Then, there is a constant $c_{3}>0$ such that $\phi(k, l) \leq c_{3} l^{s_{i} m_{i}+n_{i}}$ for every $(k, l) \in \mathbb{N}^{*} \times \mathbb{N}^{*}$ with $k \leq l^{s_{i}}$.

Proof. The first part is a consequence of (i) of Proposition 2, for $c_{1}=1+\sum_{(j, \alpha) \in\left(\Lambda_{0} \backslash\{(m, 0)\}\right)}\left|c_{j, \alpha}\right|$.

The statement (ii) is a consequence of the fact that $L(\lambda, a)$ is a polynomial of degree $m$ in $\lambda$.

In the case $1 \leq i<p$, the statement (iii) follows from Lemmas 12 and 13 given below. In the case $i=p$, then $s_{p}=0$ and $k=1$, so $\phi(k, l)=\phi(1, l) \leq c_{3} l^{n_{p}}=c_{3} k^{m_{p}} l^{n_{p}}$ for some $c_{3}>0$ (which is independent of $l$ ).

Both situations described in Lemma 12 and 13 are illustrated in Figure 3.
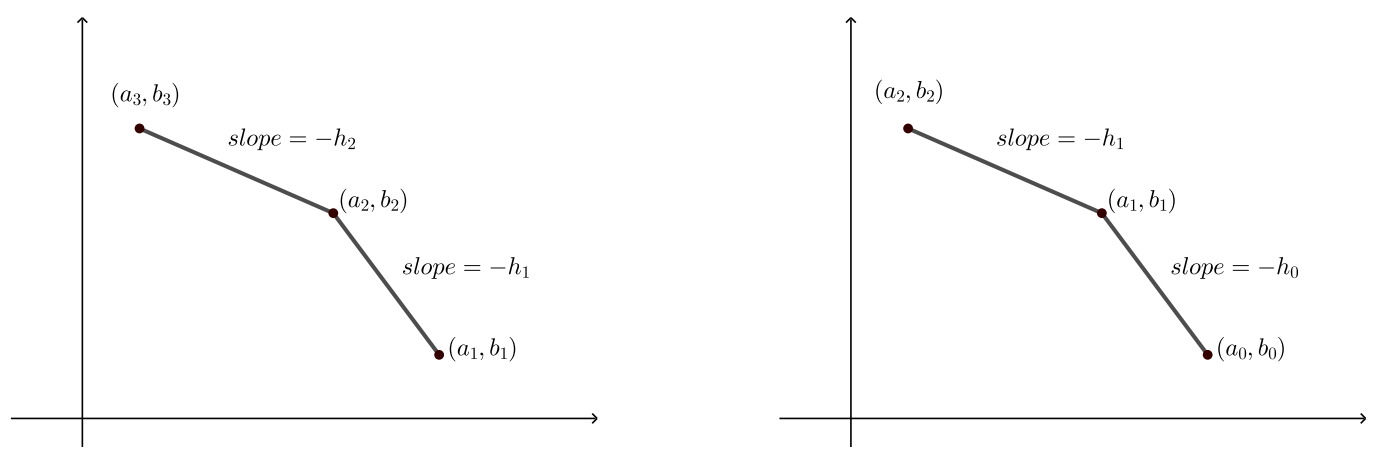

Figure 3: Geometry in Lemma 12 (left) and Lemma 13 (right)

Lemma 12. Let $0 \leq a_{3}<a_{2}<a_{1}$ and $0 \leq b_{1}<b_{2}<b_{3}$. Set $h_{1}=\left(b_{2}-b_{1}\right) /\left(a_{1}-a_{2}\right)$ and $h_{2}=\left(b_{3}-b_{2}\right) /\left(a_{2}-a_{3}\right)$. If $h_{1}>h_{2}$ holds, there is $c>0$ such that for any $(k, l) \in \mathbb{N}^{*} \times \mathbb{N}^{*}$ with $k \leq l^{h_{1}}$ we have

$$
k^{a_{1}} l^{b_{1}}+k^{a_{2}} l^{b_{2}}+k^{a_{3}} l^{b_{3}} \leq c l^{h_{1} a_{1}+b_{1}} .
$$

Proof. We set $h_{*}=\left(b_{3}-b_{1}\right) /\left(a_{1}-a_{3}\right)$. Then, $h_{1}>h_{*}$. If $k \leq l^{h_{1}}$ we have $k^{a_{1}} l^{b_{1}}+k^{a_{2}} l^{b_{2}}+k^{a_{3}} l^{b_{3}} \leq\left(l^{h_{1}}\right)^{a_{1}} l^{b_{1}}+\left(l^{h_{1}}\right)^{a_{2}} l^{b_{2}}+\left(l^{h_{1}}\right)^{a_{3}} l^{b_{3}}=\left(l^{h_{1}}\right)^{a_{1}} l^{b_{1}}\left(1+1+l^{-\left(h_{1}-h_{*}\right)\left(a_{1}-a_{3}\right)}\right)$.

Since $\left(h_{1}-h_{*}\right)\left(a_{1}-a_{3}\right)>0$, this leads us to $(38)$.

The proof of Lemma 13 is analogous to that of Lemma 12, so we omit it. 
Lemma 13. Let $0 \leq a_{2}<a_{1}<a_{0}$ and $0 \leq b_{0}<b_{1}<b_{2}$. Set $h_{0}=\left(b_{1}-b_{0}\right) /\left(a_{0}-a_{1}\right)$ and $h_{1}=\left(b_{2}-b_{1}\right) /\left(a_{1}-a_{2}\right)$. If $h_{0}>h_{1}$ holds, there is a constant $c>0$ such that

$$
k^{a_{0}} l^{b_{0}}+k^{a_{1}} l^{b_{1}}+k^{a_{2}} l^{b_{2}} \leq c l^{h_{1} a_{1}+b_{1}}
$$

for every $(k, l) \in \mathbb{N}^{*} \times \mathbb{N}^{*}$ and $k \leq l^{h_{1}}$.

Lemma 14. The following statements hold:

(i) For any $a>0, b>0, c>0, d>0$ and $0 \leq \delta<1$ we have $\lim _{\mathbb{N}^{*} \ni l \rightarrow \infty} \frac{l !^{a}}{\left[c l^{\delta}+d\right] !^{b}}=\infty$.

(ii) For $a>b \geq 0, c>0$ and $0 \leq \delta<1$ we have $\lim _{\mathbb{N}^{*} \ni l \rightarrow \infty} \frac{l !^{a}}{\left[c l^{\delta}+l\right] !^{b}}=\infty$.

Proof. Since $\left[c l^{\delta}+d\right] \leq c l^{\delta}+d \leq(c+d) l^{\delta}$ holds, to show (i) it is enough to prove

$$
\lim _{x \rightarrow \infty} \frac{\Gamma(x+1)^{a}}{\Gamma\left(c_{1} x^{\delta}+1\right)^{b}}=\infty, \quad \text { that is, } \quad \lim _{x \rightarrow \infty} \log \left(\frac{\Gamma(x+1)^{a}}{\Gamma\left(c_{1} x^{\delta}+1\right)^{b}}\right)=\infty,
$$

where $c_{1}=c+d$. This is a direct consequence of Stirling's formula. The second part of the proof is attained by analogous arguments.

\subsection{Proof of Lemma 10}

Let $L(\lambda, \rho)$ be as in (4). By setting $p=p_{h, \beta}$ and $q=i+|\boldsymbol{\nu}|-1$, we can write the equation (37) as follows:

$$
L\left(t \partial_{t}, x \partial_{x}\right) u=A x^{m} t+B x^{p}\left(t \partial_{t}\right)^{h}\left[x \partial_{x}\right]_{\beta} u+C t^{q} x^{\mu}\left(t \partial_{t}\right)^{j} \partial_{x}^{\alpha} u
$$

where $\left[x \partial_{x}\right]_{0}=1$ and $\left[x \partial_{x}\right]_{\beta}=\left(x \partial_{x}\right)\left(x \partial_{x}-1\right) \cdots\left(x \partial_{x}-\beta+1\right)$ for $\beta \geq 1$. For the sake of clarity, we summarize the main hypotheses on (39):

$$
\begin{array}{ll}
\left.\mathrm{h}_{1}\right) \quad A>0, B>0, C>0 ; & \left.\mathrm{h}_{3}\right)(h, \beta) \in I_{m} \text { and }(h, \beta) \notin \mathcal{N}_{0} ; \\
\left.\mathrm{h}_{2}\right) \quad p, q, \mu \in \mathbb{N} \text {, satisfy } p \geq 1, q \geq 1 ; & \left.\mathrm{h}_{4}\right)(j, \alpha) \in I_{m} .
\end{array}
$$

Since $(h, \beta) \in I_{m}$, we have $0 \leq h<m$ and so we can find an $i \in\{1, \ldots, p\}$ such that $m_{i+1} \leq$ $h<m_{i}$ holds. We set $d=d_{h, \beta}$. Then, $d=\min \left\{y \in \mathbb{R} ;(h, \beta-y) \in \mathcal{N}_{0}\right\}=\beta-n_{i}-s_{i}\left(m_{i}-h\right)$. Since $(h, \beta) \notin \mathcal{N}_{0}$ we have $d>0$. The situation is illustrated in Figure 4. Since $(h, \beta) \in I_{m}$ and $(h, \beta) \notin \mathcal{N}_{0}$ we have $0 \leq s_{i}<1$. We set

$$
\sigma_{0}^{*}=1+\frac{d}{p}, \quad s_{0}^{*}=1+\max \left[0, \frac{j+\alpha+(d / p)(\alpha-\mu)-m}{q}\right] .
$$

Then, Lemma 10 is stated in the following form:

Proposition 4. The equation (39) has a unique formal solution $u(t, x) \in \mathbb{C}[[t, x]]$ satisfying $u(0, x) \equiv 0$, and it belongs to the class $G\{t, x\}_{(s, \sigma)}$ if and only if $(s, \sigma)$ satisfies $s \geq s_{0}^{*}$ and $\sigma \geq \sigma_{0}^{*}$.

Proof. We note that if $\mu \geq \alpha$, we have $s_{0}^{*}=1$. As is seen in the first part of Section $5, L(\lambda, \rho)$ satisfies (N) and (GP); therefore, the sufficiency follows from Theorem 2. Our purpose is to show the necessity of the condition: $s \geq s_{0}^{*}$ and $\sigma \geq \sigma_{0}^{*}$. We will show this in the cases $\mu<\alpha$ and $\mu \geq \alpha$ separately. 


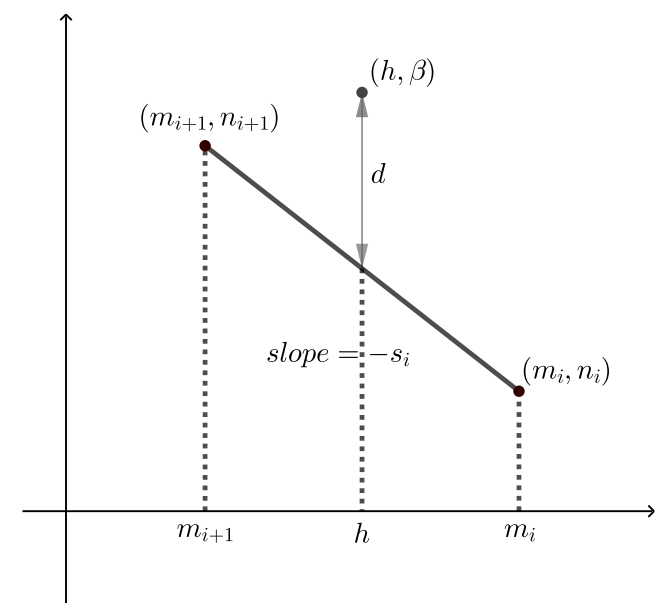

Figure 4: Geometry related to equation (39)

Case $\mu<\alpha$ :

Now, we suppose that $u(t, x) \in G\{t, x\}_{(s, \sigma)}$ holds for some $s \geq 1$ and $\sigma \geq 1$. Let us show that $s \geq s_{0}^{*}$ and $\sigma \geq \sigma_{0}^{*}$ hold, in different steps.

In the discussion below, for two sequences of positive numbers $\left\{A_{l} ; l \in \mathbb{N}^{*}\right\}$ and $\left\{B_{l} ; l \in \mathbb{N}^{*}\right\}$ we write $A_{l} \gtrsim B_{l}$ if there are $M>0$ and $H>0$ such that $A_{l} \geq M H^{l} B_{l}$ holds for all $l \in \mathbb{N}^{*}$. In this case, for $\rho>0$ we also write

$$
\sum_{l \geq 1} A_{l} \rho^{l} \gtrsim \sum_{l \geq 1} B_{l} \rho^{l}
$$

By Stirling's formula we have

Lemma 15. The following statements hold:

(i) We have $l^{l} \gtrsim l$ ! and $l$ ! $l^{l}$.

(ii) For a fixed $n \in \mathbb{N}^{*}$ we have $(n l) ! \gtrsim l !^{n}$ and $l ! \gtrsim(n l) !^{1 / n}$.

(iii) For fixed $m, n \in \mathbb{N}^{*}$ we have $(n l+m)^{l} \gtrsim l !^{n}$.

Step 1. Let $u(t, x)=\sum_{k \geq 1} u_{k}(x) t^{k} \in(\mathbb{C}[[x]])[[t]]$ be a formal solution of (39). Then, the coefficients $u_{k}(x)(k=1,2, \ldots)$ are determined by the recurrence formulas

$$
\begin{gathered}
L\left(1, x \partial_{x}\right) u_{1}=A x^{m}+B x^{p}\left[x \partial_{x}\right]_{\beta} u_{1} \\
L\left(k, x \partial_{x}\right) u_{k}=B x^{p} k^{h}\left[x \partial_{x}\right]_{\beta} u_{k}+C(k-q)^{j} x^{\mu} \partial_{x}^{\alpha} u_{k-q}, \quad k \geq 2 .
\end{gathered}
$$

The function $u_{1}(x)$ is given by $u_{1}(x)=\sum_{l \geq 0} A_{0, l p+m} x^{l p+m}$ with

$$
A_{0, l p+m}=\frac{A B^{l}[m]_{\beta}[p+m]_{\beta} \cdots[(l-1) p+m]_{\beta}}{L(1, m) L(1, p+m) \cdots L(1, l p+m)}, \quad l \geq 0 .
$$

Moreover, one can check that $u(t, x)$ has the form

$$
u(t, x)=\sum_{k \geq 0} u_{k q+1}(x) t^{k q+1}
$$


and the coefficients $u_{k q+1}(x)(k=1,2, \ldots)$ are determined by the following recurrence formulas:

$$
L\left(k q+1, x \partial_{x}\right) u_{k q+1}=B x^{p}(k q+1)^{h}\left[x \partial_{x}\right]_{\beta} u_{k q+1}+C((k-1) q+1)^{j} x^{\mu} \partial_{x}^{\alpha} u_{(k-1) q+1} .
$$

Step 2. We set $d_{0}=m$, and define $\left(l_{k-1}, d_{k}\right)(k=1,2, \ldots)$ by

$$
l_{k-1}=\min \left\{l \in \mathbb{N}: l p+d_{k-1} \geq m+\alpha-\mu\right\}, \quad d_{k}=l_{k-1} p+d_{k-1}-\alpha+\mu,
$$

inductively on $k$.

Lemma 16. For any $k \in \mathbb{N}^{*}$ we have the following results:

(i) $0 \leq l_{k-1} \leq \alpha$ and $m \leq d_{k} \leq m+p$.

(ii) $l p+d_{k-1}-\alpha+\mu=\left(l-l_{k-1}\right) p+d_{k}$.

(iii) If $C_{l p+d_{k-1}} \geq 0$ holds for all $l \in \mathbb{N}$, we have

$$
x^{\mu} \partial_{x}^{\alpha} \sum_{l \geq 0} C_{l p+d_{k-1}} x^{l p+d_{k-1}} \gg \sum_{l \geq 0} C_{l p+d_{k}+(\alpha-\mu)} x^{l p+d_{k}} \gg C_{d_{k}+(\alpha-\mu)} x^{d_{k}}=C_{l_{k-1} p+d_{k-1}} x^{d_{k}} .
$$

Proof. Since $\alpha p+d_{0} \geq \alpha+m \geq \alpha+m-\mu$ holds, we have $l_{0} \leq \alpha$. By the definition of $d_{1}$ we have $m \leq d_{1} \leq m+p$. Let us show the general case of (i) by induction on $k$. Let $k \geq 2$ and suppose that $0 \leq l_{k-2} \leq \alpha$ and $m \leq d_{k-1} \leq m+p$ are known. Since $\alpha p+d_{k-1} \geq \alpha+m \geq \alpha+m-\mu$ holds, we have $l_{k-1} \leq \alpha$. By the definition of $d_{k}$, and taking into account that $\alpha>\mu$ we get

$$
m \leq d_{k} \leq m+\max \left\{d_{k-1}-(m+\alpha-\mu), p\right\} \leq m+\max \{p-(\alpha-\mu), p\}=m+p,
$$

which entails (i).

The result (ii) is clear from the definition of $d_{k}$.

In view of the second statement, and since $l_{k-1} p+d_{k-1}=d_{k}+\alpha-\mu \geq m+\alpha-\mu>\alpha$ holds, (iii) follows from the fact that

$$
\begin{aligned}
& x^{\mu} \partial_{x}^{\alpha} \sum_{l \geq 0} C_{l p+d_{k-1}} x^{l p+d_{k-1}}=\sum_{\substack{l \geq 0 \\
\left(l p+d_{k-1} \geq \alpha\right)}} C_{l p+d_{k-1}} \frac{\left(l p+d_{k-1}\right) !}{\left(l p+d_{k-1}-\alpha\right) !} x^{l p+d_{k-1}-\alpha+\mu} \\
& \gg \sum_{l \geq l_{k-1}} C_{l p+d_{k-1}} x^{l p+d_{k-1}-\alpha+\mu} \gg C_{d_{k}+(\alpha-\mu)} x^{d_{k}}=C_{l_{k-1} p+d_{k-1}} x^{d_{k}} .
\end{aligned}
$$

Step 3. We set $w_{0}(x)=u_{1}(x)$. By (iii) of Lemma 16 we have $C x^{\mu} \partial_{x}^{\alpha} w_{0} \gg C A_{0, l_{0} p+m} x^{d_{1}}=$ $K_{1} x^{d_{1}}$ with $K_{1}=C A_{0, l_{0} p+m}$. Let us define $w_{1}(x)$ by the solution of

$$
L\left(q+1, x \partial_{x}\right) w_{1}=B x^{p}(q+1)^{h}\left[x \partial_{x}\right]_{\beta} w_{1}+K_{1} x^{d_{1}} .
$$

Then we have $u_{q+1}(x) \gg w_{1}(x)=\sum_{l \geq 0} A_{1, l p+d_{1}} x^{l p+d_{1}}$, where

$$
A_{1, l p+d_{1}}=\frac{K_{1} B^{l}(q+1)^{h l}\left[d_{1}\right]_{\beta}\left[p+d_{1}\right]_{\beta} \cdots\left[(l-1) p+d_{1}\right]_{\beta}}{L\left(q+1, d_{1}\right) L\left(q+1, p+d_{1}\right) \cdots L\left(q+1, l p+d_{1}\right)}, \quad l \geq 0 .
$$

Regarding (iii) of Lemma 16 we derive $C(q+1)^{j} x^{\mu} \partial_{x}^{\alpha} w_{1} \gg C(q+1)^{j} A_{1, l_{1} p+d_{1}} x^{d_{2}}=K_{2} x^{d_{2}}$ with $K_{2}=C(q+1)^{j} A_{1, l_{1} p+d_{1}}$. 
The construction follows recursively. Assume $w_{k-1}(x)=\sum_{l>0} A_{k-1, l p+d_{k-1}} x^{l p+d_{k-1}}$. By setting $K_{k}=C((k-1) q+1)^{j} A_{k-1, l_{k-1} p+d_{k-1}}$ and defining $w_{k}(x)$ by the solution of

$$
L\left(k q+1, x \partial_{x}\right) w_{k}=B x^{p}(k q+1)^{h}\left[x \partial_{x}\right]_{\beta} w_{k}+K_{k} x^{d_{k}},
$$

then we have $u_{k q+1}(x) \gg w_{k}(x)$ and $w_{k}(x)=\sum_{l \geq 0} A_{k, l p+d_{k}} x^{l p+d_{k}}$, where

$$
A_{k, l p+d_{k}}=\frac{K_{k} B^{l}(k q+1)^{h l}\left[d_{k}\right]_{\beta}\left[p+d_{k}\right]_{\beta} \cdots\left[(l-1) p+d_{k}\right]_{\beta}}{L\left(k q+1, d_{k}\right) L\left(k q+1, p+d_{k}\right) \cdots L\left(k q+1, l p+d_{k}\right)}, \quad l \geq 0 .
$$

Step 4. By the discussion in Step 3 we have the following result. We can define $\left(K_{k}, A_{k, l p+d_{k}}\right)$ $\left(k \in \mathbb{N}\right.$ and $l \in \mathbb{N}$ ) inductively on $k: K_{0}=A, A_{0, l p+d_{0}}$ as in (40), and for $k \geq 1$ we set $K_{k}=C((k-1) q+1)^{j} A_{k-1, l_{k-1} p+d_{k-1}}$ and $A_{k, l p+d_{k}}$ as in (43). We define

$$
w(t, x)=\sum_{k \geq 0, l \geq 0} A_{k, l p+d_{k}} t^{k q+1} x^{l p+d_{k}} .
$$

Then we have $u(t, x) \gg w(t, x)$.

Lemma 17. In the previous situation, the following statements hold:

(i) There are $C_{1}>0$ and $H_{1}>0$ such that

$$
K_{k} \geq C_{1} H_{1}{ }^{k} \frac{1}{k !^{m(\alpha+1)}} \quad \text { for any } k=0,1,2, \ldots
$$

(ii) Let $s_{i}$ be as in Figure 4. For $l \in \mathbb{N}^{*}$ we set $k_{l}=\left[\left((l p+m+p)^{s_{i}}-1\right) / q\right]$. Then, there are $C_{2}>0, H_{2}>0, a>0$ and $b>0$ such that

$$
A_{k_{l}, l p+d_{k_{l}}} \geq C_{2} H_{2}{ }^{l} \frac{l !^{d}}{\left[a l^{s_{i}}+b\right] !^{m(\alpha+1)}} \quad \text { for any } l \in \mathbb{N}^{*} .
$$

Proof. The definition of $K_{k}, A_{k, l_{k} p+d_{k}}$ entails

$$
K_{k+1}=C(k q+1)^{j} A_{k, l_{k} p+d_{k}} \geq \frac{K_{k} C B^{l_{k}}}{L\left(k q+1, l_{k} p+d_{k}\right)^{l_{k}+1}} .
$$

Since $0 \leq l_{k} \leq \alpha$, we have $B^{l_{k}} \geq b_{1}(k \in \mathbb{N})$ for some $b_{1}>0$. Since $l_{k} p+d_{k} \leq \alpha p+m+p$, by (ii) of Lemma 11 we have $L\left(k q+1, l_{k} p+d_{k}\right) \leq b_{2}(k+1)^{m}(k \in \mathbb{N})$ for some $b_{2} \geq 1$. Therefore,

$$
K_{k+1} \geq \frac{K_{k} C b_{1}}{\left(b_{2}(k+1)^{m}\right)^{l_{k}+1}} \geq \frac{K_{k} C b_{1}}{\left(b_{2}(k+1)^{m}\right)^{\alpha+1}}
$$

for any $k \in \mathbb{N}$. Since $K_{0}=A$, the previous inequality leads us to (44).

Let us show the second statement. By (43), (44) and (i) of Lemma 11 we have

$$
A_{k, l p+d_{k}} \geq \frac{C_{1} H_{1}{ }^{k}}{k !^{m(\alpha+1)}} \times \frac{B^{l}(k q+1)^{h l} l !^{\beta}}{L\left(k q+1, l p+d_{k}\right)^{l+1}} \geq \frac{C_{1} H_{1}{ }^{k}}{k !^{m(\alpha+1)}} \times \frac{B^{l}(k q+1)^{h l} l !^{\beta}}{\left(c_{1} \phi(k q+1, l p+m+p)\right)^{l+1}}
$$

Since $k_{l}=\left[\left((l p+m+p)^{s_{i}}-1\right) / q\right]$, we have $k_{l} q+1 \leq(l p+m+p)^{s_{i}} \leq l p+m+p$ and so by (iii) of Lemma 11 we have

$$
\phi\left(k_{l} q+1, l p+m+p\right) \leq c_{3}(l p+m+p)^{s_{i} m_{i}+n_{i}}, \quad l \in \mathbb{N}^{*}
$$


for some $c_{3}>0$.

Similarly, we have $H_{1}{ }^{k_{l}} \geq\left(\min \left\{1, H_{1}\right\}\right)^{(l p+m+p-1) / q}$. Since $0 \leq s_{i}<1$, we have $(l p+m+$ $p)^{s_{i}} \leq(l p)^{s_{i}}+(m+p)^{s_{i}}$ and so by setting $a=p^{s_{i}} / q$ and $b=\left((m+p)^{s_{i}}-1\right) / q$ we have $k_{l} \leq\left[a l^{s_{i}}+b\right]$.

Since $(l p+m+p)^{s_{i}} \geq 1$ we have $k_{l} \geq 0$ and so $k_{l} q+1 \geq 1$. Taking into account the previous statements, and $k_{l} q+1 \geq(l p+m+p)^{s_{i}}-q$, we derive

$$
k_{l} q+1 \geq \max \left\{1,(l p+m+p)^{s_{i}}-q\right\} \geq \max \left\{1,(l p)^{s_{i}}-q\right\} \geq \frac{p^{s_{i}}}{q+1} l^{s_{i}} .
$$

In the last inequality we have used the fact that $\max \{1, x-q\} \geq x /(q+1)$ for any $x \in \mathbb{R}$.

Thus, by applying these estimates to (46), under the condition $k_{l}=\left[\left((l p+m+p)^{s_{i}}-1\right) / q\right]$ we have

$$
\begin{aligned}
A_{k_{l}, l p+d_{k_{l}}} & \geq \frac{C_{1}\left(\min \left\{1, H_{1}\right\}\right)^{(l p+m+p-1) / q}}{\left[a l^{s_{i}}+b\right] !^{m(\alpha+1)}} \frac{B^{l}\left(p^{s_{i}} /(q+1)\right)^{h l}\left(l^{s_{i}}\right)^{h l} l !^{\beta}}{\left(c_{0} c_{3}(l p+m+p)^{s_{i} m_{i}+n_{i}}\right)^{l+1}} \\
& \gtrsim \frac{l !^{s_{i} h} l !^{\beta}}{\left[a l^{s_{i}}+b\right] !^{m(\alpha+1)} \times l !^{s_{i} m_{i}+n_{i}}}=\frac{l !^{d}}{\left[a l^{s_{i}}+b\right] !^{m(\alpha+1)}} .
\end{aligned}
$$

In the above, we have used that $d=\beta-n_{i}-s_{i}\left(m_{i}-h\right)$. This proves (45).

Step 5. Let us show the condition: $\sigma \geq \sigma_{0}^{*}$. Since $u(t, x) \in G\{t, x\}_{(s, \sigma)}$ is supposed and since $u(t, x) \gg w(t, x)$ is known, we have $w(t, x) \in G\{t, x\}_{(s, \sigma)}$, that is,

$$
\sum_{k \geq 0, l \geq 0} \frac{A_{k, l p+d_{k}}}{(k q+1) !^{-1}\left(l p+d_{k}\right) !^{\sigma-1}} \rho^{k q+1} \rho^{l p+d_{k}}<\infty
$$

holds for some $0<\rho \leq 1$.

If we set $k_{l}=\left[\left((l p+m+p)^{s_{i}}-1\right) / q\right]$ we have $k_{l} q+1 \leq(l p+m+p)^{s_{i}} \leq(l p)^{s_{i}}+(m+p)^{s_{i}}=$ $a_{1} l^{s_{i}}+b_{1}$ with $a_{1}=p^{s_{i}}$ and $b_{1}=(m+p)^{s_{i}}$. Therefore, by (ii) of Lemma 17 we have

$$
\begin{aligned}
& \infty>\sum_{l \geq 1, k_{l}=\left[\left((l p+m+p)^{s_{i}}-1\right) / q\right]} \frac{A_{k_{l}, l p+d_{k_{l}}} \rho^{k_{l} q+1} \rho^{l p+d_{k_{l}}}}{\left(k_{l} q+1\right) !^{s-1}\left(l p+d_{k_{l}}\right) !^{\sigma-1}} \\
& \geq \sum_{l \geq 1} \frac{C_{2} H_{2}{ }^{l} l !^{d} \rho^{l p+m+p} \rho^{l p+m+p}}{\left[a l^{s_{i}}+b\right] !^{m(\alpha+1)}\left[a_{1} l^{s_{i}}+b_{1}\right] !^{s^{-1}}(l p+m+p) !^{\sigma-1}} \\
& \gtrsim \sum_{l \geq 1} \frac{l !^{d} \rho^{l p+m+p} \rho^{l p+m+p}}{\left[a l^{s_{i}}+b\right] !^{m(\alpha+1)}\left[a_{1} l^{s_{i}}+b_{1}\right] !^{! s-1} l !^{p(\sigma-1)}} .
\end{aligned}
$$

If $d>p(\sigma-1)$ holds, we can derive a contradiction in the following way: if we set $2 \epsilon=$ $d-p(\sigma-1)>0$, by (47) and (i) of Lemma 14 we have

$$
\infty>\sum_{l \geq 1} \frac{l !^{\epsilon}}{\left[a l^{s_{i}}+b\right] !^{m(\alpha+1)}\left[a_{1} l^{s_{i}}+b_{1}\right] !^{s-1}} l !^{\epsilon} \rho_{1}^{l} \geq C_{1} \sum_{l \geq 1} l !^{\epsilon} \rho_{1}^{l}=\infty
$$

for some $0<\rho_{1}<\rho$ and some $C_{1}>0$. Then, $\sigma \geq 1+d / p=\sigma_{0}^{*}$.

Step 6. We express every coefficient of $u(t, x)$ in (41) in the form

$$
u_{k q+1}(x)=\sum_{l \geq 0} u_{k q+1, l} x^{l}
$$


By (42) we have $L\left(k q+1, x \partial_{x}\right) u_{k q+1} \gg C((k-1) q+1)^{j} x^{\mu} \partial_{x}^{\alpha} u_{(k-1) q+1}$, which entails

$$
u_{k q+1, l} \geq \frac{C((k-1) q+1)^{j}(l-\mu+1)^{\alpha}}{L(k q+1, l)} u_{(k-1) q+1,(\alpha-\mu)+l}, \quad l \geq \mu .
$$

Hence, by using this estimate $l p$-times and by the estimate $u_{k q+1}(x) \gg w_{k}(x)$ we have

$$
\begin{aligned}
u_{(k+l p) q+1, d_{k}} & \geq \frac{C^{l p} \prod_{n=0}^{l p-1}((k+n) q+1)^{j} \prod_{n=0}^{l p-1}\left(n(\alpha-\mu)+d_{k}-\mu+1\right)^{\alpha}}{\prod_{n=0}^{l p-1} L\left((k+l p-n) q+1, n(\alpha-\mu)+d_{k}\right)} u_{k q+1, l p(\alpha-\mu)+d_{k}} \\
& \geq \frac{C^{l p}(l p) ! j(l p) !^{\alpha}}{L\left((k+l p) q+1, l p(\alpha-\mu)+d_{k}\right)^{l p}} A_{k, l p(\alpha-\mu)+d_{k}} \\
& \geq \frac{C^{l p}(l p) !^{j}(l p) !^{\alpha}}{\left(c_{1} \phi\left((k+l p) q+1, l p(\alpha-\mu)+d_{k}\right)\right)^{l p}} A_{k, l p(\alpha-\mu)+d_{k}} .
\end{aligned}
$$

Here, we set $k_{l}=\left[\left((l p(\alpha-\mu)+m+p)^{s_{i}}-1\right) / q\right]\left(l \in \mathbb{N}^{*}\right)$. Since $m_{r}+n_{r} \leq m(r=1, \ldots, p)$ hold, we have

$\phi\left(\left(k_{l}+l p\right) q+1, l p(\alpha-\mu)+d_{k_{l}}\right) \leq \sum_{r=1}^{p}\left((l p(\alpha-\mu)+m+p)^{s_{i}}+l p q\right)^{m_{r}}(l p(\alpha-\mu)+m+p)^{n_{r}} \leq c_{3}(l p)^{m}$,

for $l \in \mathbb{N}^{*}$, for some $c_{3}>0$. Therefore, under the condition $k_{l}=\left[\left((l p(\alpha-\mu)+m+p)^{s_{i}}-1\right) / q\right]$, by applying (49) and (45) to (48) we have

$$
\begin{aligned}
u_{\left(k_{l}+l p\right) q+1, d_{k_{l}}} & \geq \frac{C^{l p}(l p) !^{j}(l p) !^{\alpha}}{\left(c_{1} c_{3}(l p)^{m}\right)^{l p}} \times C_{2} H_{2}{ }^{l(\alpha-\mu)} \frac{(l(\alpha-\mu)) !^{d}}{\left[a(l(\alpha-\mu))^{s_{i}}+b\right] !^{m(\alpha+1)}} \\
& \gtrsim \frac{(l p) !^{j}(l p) !^{\alpha}}{(l p) !^{m}} \frac{(l p) !^{!^{\prime}(d p)(\alpha-\mu)}}{\left[a_{0}(l p)^{s_{i}}+b\right] !^{m(\alpha+1)}}
\end{aligned}
$$

where $a_{0}=a((\alpha-\mu) / p)^{s_{i}}$. Thus, by setting $K=j+\alpha+(d / p)(\alpha-\mu)-m$ and $k_{l}=[((l p(\alpha-$ $\left.\left.\mu)+m+p)^{s_{i}}-1\right) / q\right]$ we have

$$
u_{\left(k_{l}+l p\right) q+1, d_{k_{l}}} \gtrsim \frac{(l p) !^{K}}{\left[a_{0}(l p)^{s_{i}}+b\right] !^{m(\alpha+1)}}, \quad l \in \mathbb{N}^{*} .
$$

Step 7. Let us write $k_{l}=\left[\left((l p(\alpha-\mu)+m+p)^{s_{i}}-1\right) / q\right]$ (for $\left.l \in \mathbb{N}^{*}\right)$. Then, it is straight that $l_{1} \neq l_{2}$ implies $k_{l_{1}}+l_{1} p \neq k_{l_{2}}+l_{2} p$.

Step 8. Lastly, by using (50) let us show the condition $s \geq s_{0}^{*}$. Since $u(t, x) \in G\{t, x\}_{(s, \sigma)}$ is supposed, there is a $0<\rho \leq 1$ such that

$$
\sum_{k \geq 0, l \geq 0} \frac{u_{k q+1, l}}{(k q+1) !^{s-1} l ! !^{\sigma-1}} \rho^{k q+1} \rho^{l}<\infty .
$$

Therefore, by (50) and Step 7 we have

$$
\begin{aligned}
\infty & >\sum_{l \geq 1, k=k_{l}} \frac{u_{(k+l p) q+1, d_{k}}}{((k+l p) q+1) !^{s-1} d_{k} !^{\sigma-1}} \rho^{(k+l p) q+1} \rho^{d_{k}} \\
& \gtrsim \sum_{l \geq 1} \frac{(l p) !^{K}}{\left[a_{0}(l p)^{s_{i}}+b\right] !^{m(\alpha+1)}\left(\left(k_{l}+l p\right) q+1\right) !^{s-1} d_{k} !^{\sigma-1}} \rho^{\left(k_{l}+l p\right) q+1} \rho^{d_{k}} .
\end{aligned}
$$


We have

$$
\begin{aligned}
\left(\left(k_{l}+l p\right) q+1\right) & =\left(k_{l} q+1\right)+l p q \leq(l p(\alpha-\mu)+m+p)^{s_{i}}+l p q \\
& \leq(l p(\alpha-\mu))^{s_{i}}+(m+p)^{s_{i}}+l p q \leq a_{2}(l p q)^{s_{i}}+l p q \leq\left(a_{2}+1\right)(l p q),
\end{aligned}
$$

with $a_{2}=((\alpha-\mu) / q)^{s_{i}}+(m+p)^{s_{i}}$. Hence, by taking a smaller $0<\rho_{1}<\rho$ we have

$$
\infty>\sum_{l \geq 1} \frac{(l p q) !^{K / q}}{\left[a_{0}(l p)^{s_{i}}+b\right] !^{m(\alpha+1)}\left[a_{2}(l p q)^{s_{i}}+l p q\right] !^{s-1}} \rho_{1}{ }^{\left(a_{2}+1\right) l p q} .
$$

If $K / q>(s-1)$, we derive a contradiction. More precisely, set $\epsilon=(K / q-(s-1)) / 3$, by $(51)$ and Lemma 14 we have

$$
\begin{aligned}
\infty & >\sum_{l \geq 1} \frac{(l p q) !^{\epsilon}}{\left[a_{0}(l p)^{s_{i}}+b\right] !^{m(\alpha+1)}} \frac{(l p q) !^{\epsilon+(s-1)}}{\left[a_{2}(l p q)^{s_{i}}+l p q\right] !^{!-1}} \times(l p q) !^{\epsilon} \rho_{1}{ }^{\left(a_{2}+1\right) l p q} \\
& \geq C_{2} \sum_{l \geq 1}(l p q) !^{\epsilon} \rho_{1}{ }^{\left(a_{2}+1\right) l p q}=\infty
\end{aligned}
$$

for some $C_{2}>0$. This entails that $s \geq s_{0}^{*}$, and completes the proof of Proposition 4 , in the case that $\mu<\alpha$.

\section{Case $\mu \geq \alpha$ :}

Suppose that $u(t, x) \in G\{t, x\}_{(s, \sigma)}$ for some $s \geq 1$ and $\sigma \geq 1$. Then, it is straight that $s \geq s_{0}^{*}=1$. We now prove that $\sigma \geq \sigma_{0}^{*}$.

Let $l_{k}=0$ and $d_{k}=m+k(\mu-\alpha)$ for $k \in \mathbb{N}$. We determine $\left(K_{k}, A_{k, l p+d_{k}}\right)$ for $(k, l) \in \mathbb{N} \times \mathbb{N}$ as in the case $\mu<\alpha$. The difference in this framework lies on the following: (i) if $\mu>\alpha$, then $d_{k} \rightarrow \infty$ as $k \rightarrow \infty$, and (ii) $K_{k}$ is determined by $K_{0}=A$ and the recurrence formula

$$
K_{k+1}=C(k q+1)^{j} A_{k, d_{k}}=C(k q+1)^{j} \frac{K_{k}}{L\left(k q+1, d_{k}\right)}, \quad k \in \mathbb{N} .
$$

Assume that Lemma 18 below holds. Then, one can show that $\sigma \geq \sigma^{*}$ analogously to Step 5 in the proof of the case $\mu<\alpha$. Hence, the proof is concluded if the following result is proved.

Lemma 18. In the previous situation, the following statements hold:

(1) There exist $C_{1}, H_{1}>0$ such that

$$
K_{k} \geq C_{1} H_{1}^{k} \frac{1}{k !^{m}}, \quad k \in \mathbb{N} .
$$

(2) Let $s_{i}$ be the slopes described in Figure 4. For all $l \in \mathbb{N}^{*}$, we set $k_{l}=\left[\left((l p+m)^{s_{i}}-1\right) / q\right]$. Then, there exist $C_{2}, H_{2}, a, b>0$ such that

$$
A_{k_{l}, l p+d_{k_{l}}} \geq C_{2} H_{2}^{l} \frac{l !^{d}}{\left[a l^{s_{i}}+b\right] !^{m}}, \quad l \in \mathbb{N}^{*} .
$$

Proof. Since $d_{k}=m+k(\mu-\alpha)$ holds, we have $L\left(k q+1, d_{k}\right)$ is a polynomial of degree $m$ with respect to $k$, and so we have $L\left(k q+1, d_{k}\right) \leq b_{2}(k+1)^{m}$, for all $k \in \mathbb{N}$ and some $b_{2} \geq 1$. Therefore, by the recurrence formula, we get

$$
K_{k+1} \geq C \frac{K_{k}}{L\left(k q+1, d_{k}\right)} \geq K_{k} \frac{C}{b_{2}(k+1)^{m}}, \quad k \in \mathbb{N},
$$


which entails (52). We now give proof for the second statement in this lemma. Following analogous arguments as in (46) we get

$$
A_{k, l p+d_{k}} \geq \frac{C_{1} H_{1}^{k}}{k !^{m}} \frac{B^{l}(k q+1)^{h l} l !^{\beta}}{\left(c_{1} \phi(k q+1, l p+m+k(\mu-\alpha))\right)^{l+1}} .
$$

The definition of $k_{l}$ yields $k_{l} q+1 \leq(l p+m)^{s_{i}} \leq\left(l p+m+k_{l}(\mu-\alpha)\right)^{s_{i}}$ and

$$
l p+m+k_{l}(\mu-\alpha) \leq l p+m+\left[\left((l p+m)^{s_{i}}\right) / q\right](\mu-\alpha) \leq b_{3} l, \quad l \in \mathbb{N}^{*}
$$

for some $b_{3}>0$. Statement (3) in Lemma 11 yields

$$
\phi\left(k_{l} q+1, l p+m+k_{l}(\mu-\alpha)\right) \leq c_{3}\left(l p+m+k_{l}(\mu-\alpha)\right)^{s_{i} m_{i}+n_{i}} \leq c_{3}\left(b_{3} l\right)^{s_{i} m_{i}+n_{i}}, \quad l \in \mathbb{N}^{*},
$$

for some $c_{3}>0$. Similarly to the case $\mu<\alpha$, one has

$$
\begin{aligned}
& H_{1}^{k_{l}} \geq\left(\min \left\{1, H_{1}\right\}\right)^{k_{l}} \geq\left(\min \left\{1, H_{1}\right\}\right)^{(l p+m-1) / q}, \\
& k_{l} \leq\left[a l^{s_{i}}+b\right], \text { for } a=p^{s_{i} / q}, b=\left(m^{s_{i}}-1\right) / q, \\
& k_{l} q+1 \geq \frac{p^{s_{i}}}{q+1} l^{s_{i}} .
\end{aligned}
$$

The application of the previous estimates on (54) with $k_{l}=\left[\left((l p+m)^{s_{i}}-1\right) / q\right]$, and taking into account that $d=\beta-n_{i}-s_{i}\left(m_{i}-h\right)$ yields to the conclusion:

$$
\begin{aligned}
A_{k_{l}, l p+d_{k_{l}}} & \geq \frac{C_{1}\left(\min \left\{1, H_{1}\right\}\right)^{(l p+m-1) / q}}{\left[a l^{s_{i}}+b\right] !^{m}} \frac{B^{l}\left(p^{s_{i}} /(q+1)\right)^{h l}\left(l^{s_{i}}\right)^{h l} l !^{\beta}}{\left(c_{0} c_{3}\left(b_{3} l\right)^{s_{i} m_{i}+n_{i}}\right)^{l+1}} \\
& \gtrsim \frac{l !^{s_{i} h} l !^{\beta}}{\left[a l^{s_{i}}+b\right] !^{m} l !^{s_{i} m_{i}+n_{i}}}=\frac{l !^{d}}{\left[a l^{s_{i}}+b\right] !^{m}} .
\end{aligned}
$$

In the following, we state a variant of Proposition 4. Let us consider the equation

$$
L\left(t \partial_{t}, x \partial_{x}\right) u=A x t+B x^{p}\left(t \partial_{t}\right)^{h}\left(x \partial_{x}\right)^{\beta} u+C t^{q} x^{\mu}\left(t \partial_{t}\right)^{j} \partial_{x}^{\alpha} u
$$

under the same assumptions $\left.\mathrm{h}_{1}\right) \sim \mathrm{h}_{4}$ ) as in (39). Let $\sigma_{0}^{*}$ and $s_{0}^{*}$ be as in Proposition 4. Then, an analogous argument as above, the next result is attained.

Proposition 5. The equation (55) has a unique formal solution $u(t, x) \in \mathbb{C}[[t, x]]$ satisfying $u(0, x) \equiv 0$, and it belongs to the class $G\{t, x\}_{(s, \sigma)}$ if and only if $(s, \sigma)$ satisfies $s \geq s_{0}^{*}$ and $\sigma \geq \sigma_{0}^{*}$

\section{A generalization}

Let $C(x ; \lambda, \rho)$ be as in (3), $\mathcal{M}$ be a finite subset of $\mathbb{N} \times \mathbb{N}$, and let $\boldsymbol{z}=\left\{z_{j, \alpha}\right\}_{(j, \alpha) \in \mathcal{M}}$ be the complex variables in $\mathbb{C}^{N}$ (with $N=\# \mathcal{M}$ ). We consider

$$
C\left(x ; t \partial_{t}, x \partial_{x}\right) u=a(x) t+G_{2}\left(t, x,\left\{\left(t \partial_{t}\right)^{j} \partial_{x}^{\alpha} u\right\}_{(j, \alpha) \in \mathcal{M}}\right),
$$


where $G_{2}(t, x, \boldsymbol{z})$ is a holomorphic function in a neighborhood of $(0,0,0) \in \mathbb{C}_{t} \times \mathbb{C}_{x} \times \mathbb{C}_{\boldsymbol{z}}^{N}$ whose Taylor expansion in $(t, \boldsymbol{z})$ has the form

$$
G_{2}(t, x, \boldsymbol{z})=\sum_{i+|\boldsymbol{\nu}| \geq 2} g_{i, \boldsymbol{\nu}}(x) t^{i} \boldsymbol{z}^{\boldsymbol{\nu}}
$$

with $\boldsymbol{\nu}=\left\{\nu_{j, \alpha}\right\}_{(j, \alpha) \in \mathcal{M}} \in \mathbb{N}^{N},|\boldsymbol{\nu}|=\sum_{(j, \alpha) \in \mathcal{M}} \nu_{j, \alpha}$ and $\boldsymbol{z}^{\boldsymbol{\nu}}=\prod_{(j, \alpha) \in \mathcal{M}} z_{j, \alpha}{ }^{\nu_{j, \alpha}}$.

If $\mathcal{M}=I_{m}$, equation (56) coincides with (1) (or (5)). We can define the irregularity $\sigma_{0}$ of (56) at $x=0$ in the same way as (8). For $\mu \in \mathbb{N}$ we set

$$
J_{\mu}=\left\{(i, \boldsymbol{\nu}) \in \mathbb{N} \times \mathbb{N}^{N} ; i+|\boldsymbol{\nu}| \geq 2,|\boldsymbol{\nu}| \geq 1,\left(\partial_{x}^{\mu} g_{i, \boldsymbol{\nu}}\right)(0) \neq 0\right\} .
$$

For $\mu \in \mathbb{N}$ and $\boldsymbol{\nu}=\left\{\nu_{j, \alpha}\right\}_{(j, \alpha) \in \mathcal{M}}$ satisfying $|\boldsymbol{\nu}| \geq 1$ we set

$$
\begin{gathered}
K_{\boldsymbol{\nu}}=\left\{(j, \alpha) \in \mathcal{M} ; \nu_{j, \alpha}>0\right\}, \quad m_{\boldsymbol{\nu}, \mu}=\max _{(j, \alpha) \in K_{\boldsymbol{\nu}}}\left(j+\max \left\{\alpha, \mu+\sigma_{0}(\alpha-\mu)\right\}\right), \\
s_{0}=1+\max \left[0, \sup _{\mu \geq 0}\left(\sup _{(i, \boldsymbol{\nu}) \in J_{\mu}} \frac{m_{\boldsymbol{\nu}, \mu}-m}{i+|\boldsymbol{\nu}|-1}\right)\right] .
\end{gathered}
$$

The same arguments as in Section 4 apply to obtain the following results.

Theorem 4. Suppose the conditions (N) and (GP) hold. Then, the equation (56) has a unique formal solution $u(t, x) \in \mathbb{C}[[t, x]]$ satisfying $u(0, x) \equiv 0$, and it belongs to the class $G\{t, x\}_{(s, \sigma)}$ for any $s \geq s_{0}$ and $\sigma \geq \sigma_{0}$.

\section{Remark.}

(i) The index $s_{0}$ is also expressed in the form

$$
s_{0}=1+\max \left[0, \sup _{\mu \geq 0}\left(\max _{(j, \alpha) \in \mathcal{M}} \frac{j+\max \left\{\alpha, \mu+\sigma_{0}(\alpha-\mu)\right\}-m}{L_{\mu, j, \alpha}}\right)\right]
$$

where $L_{\mu, j, \alpha}=\operatorname{val}\left(\left(\partial_{z_{j, \alpha}} \partial_{x}{ }^{\mu} G_{2}\right)(t, 0, \boldsymbol{z})\right)(\mu \in \mathbb{N}$ and $(j, \alpha) \in \mathcal{M})$.

(ii) If $\sigma_{0}=1$, we have

$$
s_{0}=1+\max \left[0, \sup _{\mu \geq 0}\left(\max _{(j, \alpha) \in \mathcal{M}} \frac{j+\alpha-m}{L_{\mu, j, \alpha}}\right)\right] .
$$

Hence, if $\sigma_{0}=1$ and $\mathcal{M} \subset\{(j, \alpha) ; j+\alpha \leq m\}$, we have $s_{0}=1$ and the formal power series solution $u(t, x)$ is convergent in a neighborhood of $(0,0) \in \mathbb{C}_{t} \times \mathbb{C}_{x}$.

As to the optimality, to get the same result as in Theorem 3 we need some additional condition. We set:

$$
\begin{aligned}
& \mathcal{L}=\left\{(i, \boldsymbol{\nu}): i+|\boldsymbol{\nu}| \geq 2,|\boldsymbol{\nu}| \geq 1, g_{i, \boldsymbol{\nu}}(x) \not \equiv 0\right\} \\
& M=\max \{\alpha ;(j, \alpha) \in \mathcal{M}\}, \quad \mathcal{M}_{\mu}=\{(j, \alpha) \in \mathcal{M} ; \alpha \geq \mu\}, \quad 0 \leq \mu \leq M, \\
& s_{1}=1+\max \left[0, \max _{0 \leq \mu \leq M}\left(\max _{(j, \alpha) \in \mathcal{M}_{\mu}} \frac{j+\mu+\sigma_{0}(\alpha-\mu)-m}{L_{\mu, j, \alpha}}\right)\right] .
\end{aligned}
$$

We note that if $\alpha \geq \mu$ we have $\max \left\{\alpha, \mu+\sigma_{0}(\alpha-\mu)\right\}=\mu+\sigma_{0}(\alpha-\mu)$.

In general, we have $s_{0} \geq s_{1}$. By the same argument as in Section 5 we have 
Theorem 5. Suppose the condition $s_{0}=s_{1}$. In addition, assume $\mathcal{L} \neq \emptyset$ and also the following conditions:

$c-1)\left(\partial_{x}^{m} a\right)(0)>0,\left(\partial_{x}^{\mu} a\right)(0)>0$ for $0 \leq \mu \leq M$ and $a(x) \gg 0$;

c-2) $c_{j, \alpha}(0) \leq 0$ for any $(j, \alpha) \in I_{m}$,

c-3) $c_{j, \alpha}(x)-c_{j, \alpha}(0) \gg 0$ for any $(j, \alpha) \in I_{m}$,

c-4) $g_{i, \boldsymbol{\nu}}(x) \gg 0$ for any $(i, \boldsymbol{\nu})$ with $i+|\boldsymbol{\nu}| \geq 2$.

Then, equation (56) has a unique formal solution $u(t, x) \in \mathbb{C}[[t, x]]$ satisfying $u(0, x) \equiv 0$, and it belongs to the class $G\{t, x\}_{(s, \sigma)}$ if and only if $(s, \sigma)$ satisfies $s \geq s_{0}$ and $\sigma \geq \sigma_{0}$.

In the case $s_{0}>s_{1}$, our index $s_{0}$ is not optimal in general, as it is seen in the following example.

Example: Let us consider

$$
t \partial_{t} u=x t+x\left(x \partial_{x}\right) u+t x^{3} \partial_{x}^{2} u .
$$

In this case, we have $\mathcal{M}=\{(0,2)\}, \sigma_{0}=2, s_{0}=2$ and $s_{1}=1$. Equation (57) has a unique formal solution $u(t, x) \in \mathbb{C}[[t, x]]$ satisfying $u(0, x) \equiv 0$, and it belongs to the class $G\{t, x\}_{(1,2)}$.

Proof. We set $u(t, x)=\sum_{k \geq 1} u_{k}(x) t^{k}$. Then, $u_{k}(x) \in \mathbb{C}[[x]](k=1,2, \ldots)$ are uniquely determined inductively on $k$ by the relations: $u_{1}=x+x\left(x \partial_{x}\right) u_{1}$ and for $k \geq 2$

$$
k u_{k}=x\left(x \partial_{x}\right) u_{k}+x^{3} \partial_{x}^{2} u_{k-1} .
$$

In addition, for any $k=1,2, \ldots$ we have $u_{k}(x) \gg 0$ and

$$
\mathcal{B}_{2}\left[u_{k}\right](x) \ll \frac{2^{k-1}}{(1-x)^{2 k-1}} .
$$

This proves that $u(t, x) \in G\{t, x\}_{(1,2)}$.

The proof of $(59)_{k}$ is as follows. The case $k=1$ is verified by a direct calculation. Let $k \geq 2$ and suppose that this property holds for $k-1$. Then, we have

$$
\mathcal{B}_{2}\left[x^{3} \partial_{x}^{2} u_{k-1}\right] \ll x^{2} \partial_{x} \mathcal{B}_{2}\left[u_{k-1}\right] \ll \frac{2^{k-2} x^{2}(2 k-3)}{(1-x)^{2 k-2}} \ll \frac{2^{k-2}(2 k-3)}{(1-x)^{2 k-2}} .
$$

Since $\mathcal{B}_{2}\left[x\left(x \partial_{x}\right) u_{k}\right] \ll x \mathcal{B}_{2}\left[u_{k}\right]$, by (58) and (60) we have

$$
\mathcal{B}_{2}\left[u_{k}\right] \ll \frac{1}{k-x} \mathcal{B}_{2}\left[x^{3} \partial_{x}^{2} u_{k-1}\right](x) \ll \frac{1}{k(1-x)} \frac{2^{k-2}(2 k-3)}{(1-x)^{2 k-2}} \ll \frac{2^{k-1}}{(1-x)^{2 k-1}} .
$$

This proves $(59)_{k}$.

Acknowledgements. The authors would like to thank the anonymous referee who has pointed out the illustrative example described in the Remark in Section 2.4. 


\section{References}

[1] I. Baldomá, E. Fontich, P. Martín. Gevrey estimates for one dimensional parabolic invariant manifolds of non-hyperbolic fixed points. Discrete Contin. Dyn. Syst. 37 (2017), no. 8, 4159 4190 .

[2] W. Balser, M. Yoshino, Gevrey order of formal power series solutions of inhomogeneous partial differential equations with constant coefficients. Funkcial. Ekvac. 53 (2010), no. 3, 411-434.

[3] M. S. Baouendi and C. Goulaouic, Singular nonlinear Cauchy problems. J. Differential Equations 22 (1976), 268-291.

[4] H. Chen and Z. Luo, On the holomorphic solution of non-linear totally characteristic equations with several space variables. Acta Math. Sci. Ser. B Engl. Ed. 22 (2002), 393-403.

[5] H. Chen and Z. Luo, Formal solutions for higher order nonlinear totally characteristic PDEs with irregular singularities, Geometric analysis of PDE and several complex variables, 121131, Contemp. Math., 368, Amer. Math. Soc., Providence, RI, 2005.

[6] H. Chen, Z. Luo and H. Tahara, Formal solutions of nonlinear first order totally characteristic type PDE with irregular singularity. Ann. Inst. Fourier, 51 (2001), 1599-1620.

[7] H. Chen, Z. Luo and C. Zhang, On the summability of formal solutions for a class of nonlinear singular PDEs with irregular singularity. Recent progress on some problems in several complex variables and partial differential equations, 53-64, Contemp. Math., 400, Amer. Math. Soc., Providence, RI, 2006.

[8] H. Chen and H. Tahara, On totally characteristic type non-linear partial differential equations in the complex domain, Publ. Res. Inst. Math. Sci., 35 (1999), 621-636.

[9] R. Gérard and H. Tahara, Solutions holomorphes et singulières d' équations aux dérivées partielles singulières non linéaires, Publ. Res. Inst. Math. Sci., 29 (1993), 121-151.

[10] R. Gérard and H. Tahara, Singular nonlinear partial differential equations, Aspects Math., E 28, Vieweg, 1996.

[11] R. Gérard and H. Tahara, Holomorphic and singular solutions of non-linear singular partial differential equations, II, "Structure of solutions of differential equations, Katata/Kyoto, 1995" (ed. Morimoto-Kawai), World Scientific, 1996, 135-150.

[12] R. Gontsov, I. Goryuchkina, The Maillet-Malgrange type theorem for generalized power series. Manuscripta Math. 156 (2018), no. 1-2, 171-185.

[13] G. K. Immink, On the Gevrey order of formal solutions of nonlinear difference equations. J. Difference Equ. Appl. 12 (2006), no. 7, 769-776.

[14] Z. Luo, H. Chen and C. Zhang, Exponential-type Nagumo norms and summability of formal solutions of singular partial differential equations. Ann. Inst. Fourier, 62 (2012), no. 2, 571618.

[15] N. S. Madi and M. Yoshino, Uniqueness and solvability of nonlinear Fuchsian equations. Bull. Sci. Math. 114 (1990), no. 1, 41-60. 
[16] E. Maillet, Sur les séries divergentes et les équations différentielles, Ann. Ecole Normale, Ser. 3, 20 (1903), 487-518.

[17] B. Malgrange, Sur le théorème de Maillet, Asymptotic Anal. 2 (1989), no. 1, 1-4.

[18] P. Remy, Gevrey order and summability of formal series solutions of some classes of inhomogeneous linear partial differential equations with variable coefficients. J. Dyn. Control Syst. 22 (2016), no. 4, 693-711.

[19] A. Shirai, Convergence of formal solutions of singular first order nonlinear partial differential equations of totally characteristic type. Funkcial. Ekvac. 45 (2002), 187-208.

[20] A. Shirai, Maillet type theorem for singular first order nonlinear partial differential equations of totally characteristic type. Part II. Opuscula Math. 35 (2015), no. 5, 689-712

[21] H. Tahara, Generalized Poincaré condition and convergence of formal solutions of some nonlinear totally characteristic equations, Tokyo J. Math., 39, no. 3 (2016), 863-883.

[22] H. Tahara and H.Yamane, Logarithmic singularities of solutions to nonlinear partial differential equations, J. Math. Soc. Japan, 60 (2008), 603-630.

[23] H. Tahara and H.Yamazawa, Structure of solutions of nonlinear partial differential equations of Gerard-Tahara type, Publ. Res. Inst. Math. Sci., 41 (2005), 339-373.

[24] L. Di Vizio, An ultrametric version of the Maillet-Malgrange theorem for nonlinear qdifference equations. Proc. Amer. Math. Soc. 136 (2008), no. 8, 2803-2814.

[25] E. T. Whittaker, G. N. Watson, A course of modern analysis (4th edition, reprinted), Cambridge Univ. Press, 1958.

[26] H. Yamazawa, Newton polyhedrons and a formal Gevrey space of double indices for linear partial differential equations, Funkt. Ekvac. 41 (1998), 337-345.

[27] C. Zhang, A Maillet-Malgrange theorem for q-difference-differential equations, Asymptot. Anal. 17 (1998), no. 4, 309-314. 